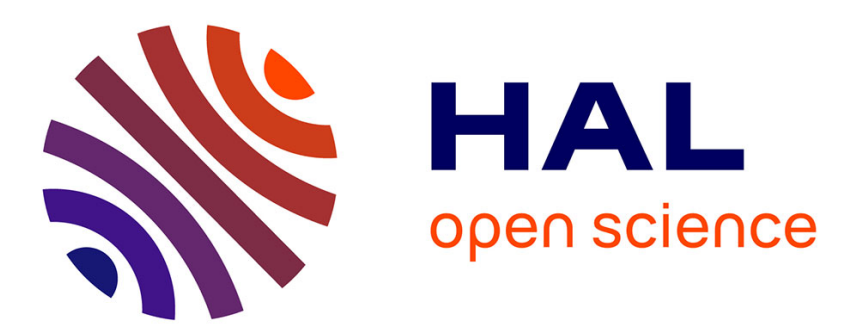

\title{
Hydro-mechanical modelling of multiphase flowin naturally fractured coalbed using a multiscale approach
}

François Bertrand, Olivier Buzzi, Pierre Bésuelle, Frédéric Collin

\section{To cite this version:}

François Bertrand, Olivier Buzzi, Pierre Bésuelle, Frédéric Collin. Hydro-mechanical modelling of multiphase flowin naturally fractured coalbed using a multiscale approach. Journal of Natural Gas Science and Engineering, 2020, 78, pp.103303. 10.1016/j.jngse.2020.103303 . hal-02558478

\section{HAL Id: hal-02558478 \\ https://hal.univ-grenoble-alpes.fr/hal-02558478}

Submitted on 22 Sep 2020

HAL is a multi-disciplinary open access archive for the deposit and dissemination of scientific research documents, whether they are published or not. The documents may come from teaching and research institutions in France or abroad, or from public or private research centers.
L'archive ouverte pluridisciplinaire HAL, est destinée au dépôt et à la diffusion de documents scientifiques de niveau recherche, publiés ou non, émanant des établissements d'enseignement et de recherche français ou étrangers, des laboratoires publics ou privés. 


\title{
Hydro-mechanical modelling of multiphase flow in naturally fractured coalbed using a multiscale approach
}

\author{
François BERTRAND ${ }^{\mathrm{a}, \mathrm{b}, \mathrm{c}}$, Olivier BuZzi ${ }^{\mathrm{b}}$, Pierre BÉSUELle ${ }^{\mathrm{d}}$, Frédéric COLLIN ${ }^{\mathrm{a}}$ \\ ${ }^{a}$ University of Liège, Urban and Environmental Engineering / Geomechanics, Liège, Belgium \\ ${ }^{b}$ University of Newcastle, Priority Research Centre for Geotechnical and Materials Modelling, Callaghan, \\ NSW, Australia \\ ${ }^{c}$ FNRS-F.R.I.A, Fonds de la Recherche Scientifique, Belgium \\ ${ }^{d}$ Grenoble Alpes, CNRS, Grenoble INP, 3SR, 38000 Grenoble, France
}

\begin{abstract}
This paper presents a multiscale model developed for the modelling of multiphase flow in fractured reservoirs, particularly in coal seams during coalbed methane recovery (or carbon dioxide storage). The variation of gas content in the matrix induces a shrinkage/swelling of the material which is likely to affect the fractures and thus the permeability of the rock. In addition, the permeability is also sensitive to the effective stress evolution. In order to simulate the reservoir production taking into account the cleat-scale phenomena on their specific length, a cleat-scale model is used for the modelling of the Representative Elementary Volume (REV at microscale). This cleat-scale model is integrated in a multiscale approach using the finite element square method. It consists to localize the macroscale deformation to the microscale by applying appropriate boundaries, then resolve the boundary value problem on the microscale with finite elements, then homogenize the microscale stresses with appropriate averages to compute macroscopic quantities, and finally resolve the boundary value problem on the macroscale with finite elements. This approach has the advantage that it does not require to write some constitutive laws at the macroscale but only at the REV-scale. The model is developed and implemented in a finite element code and then used for reservoir modelling. A synthetic case is first considered to demonstrate the ability of the model to obtain some bell-shape production curves as expected for unconventional reservoirs. Finally, a history matching exercise is carried out.
\end{abstract}

Keywords: Numerical model, unsaturated fractured medium, permeability, hydro-mechanical couplings, Langmuir, shrinkage

\section{Introduction}

Changes of coal properties during methane production (or carbon storage) are a critical issue. Indeed, ignoring geomechanical processes certainly leads to errors in the evaluation of coalbed methane (CBM) production as the permeability of the reservoir is affected (Gu et al., 2005). In existing CBM models, sorption- and stress-induced coal permeability alteration is a remarkable aspect which is often improperly simplified 
(Wei et al., 2007) and it is clear that further work is required in order to improve our understanding of the reservoir behaviour of coal permeability (Moore, 2012).

Coal is generally naturally fractured, the divisions are known as cleats. Fracturing is often most abundant in bright bands of coal (Paterson et al., 1992). Fracture spacing can range from fractions of a millimeter in vitrain layers to few centimeters or even non-apparent in durain (Dawson and Esterle, 2010). Coal rank plays also a role in the cleat development, cleat frequency increases from lignite to low-volatile bituminous coal and then decreases above that rank (Moore, 2012). This cleat network is of course very important for the permeability.

Ideally, a numerical model would detail the material microstructure with separate descriptions of each constituent, matrix blocks and cleats. This approach was followed in one of our previous paper (Bertrand et al., 2019) where a model was developed at the cleat-scale. Two major couplings are considered: the swelling/shrinkage of the material with the gas content and the cleat aperture opening/closure with the (effective) stress state.

However, direct modelling of the entire microstructure is usually not possible due to the high computational expense it would require at the scale of a reservoir. In consequence, large-scale models are generally phenomenological in nature: the behaviour of all the constituents is represented collectively by closed-form macroscopic constitutive equations. The phenomenological approach has limits because improving the macroscale models by taking into account more and more micromechanical effects makes it more and more difficult to formulate. Over the past few decades, many permeability models have been developed for coal reservoirs (Somerton et al., 1975; Schwerer et al., 1984; Seidle et al., 1992; Palmer et al., 1996; Gilman and Beckie, 2000; Lu and Connell, 2007; Connell et al., 2010; Gu and Chalaturnyk, 2010; Pan and Connell, 2012; Chen et al., 2013; Shi et al., 2014; Lu et al., 2016; Bertrand et al., 2017) to name a few, but these models are either analytical formulations or macroscopic models.

An alternative description is the use of a multi-level approach. The idea is to model the micromechanical effects explicitly on their specific length scale through a direct modelling and couple their homogenized effects to the macroscale. The physical behaviour of the material at the macroscale is derived from the modelling of a representative elementary volume (REV) which contains a detailed model of the material microstructure. The macroscopic phenomenological constitutive law is replaced by suitable averages over the REV. The analysis on the REV level can be realised using different methods: the finite element method (Terada and Kikuchi, 1995; Smit et al., 1998; Miehe et al., 1999; Feyel and Chaboche, 2000; Kouznetsova et al., 2001; Massart, 2003; Özdemir et al., 2008; Larsson et al., 2010b; Su et al., 2011; Frey et al., 2013), the discrete element method (DEM) (Nitka et al., 2011; Nguyen et al., 2014), the Voronoi cell method (Ghosh et al., 1995) or the Fourier series approach (Moulinec and Suquet, 1998). When finite elements are used at both scales, the method is called the finite element square method $\left(F E^{2}\right)$ (Kouznetsova et al., 2001). We employ this method in this paper to develop a double-scale model describing the coal behaviour.

The $F E^{2}$ method is based on the following consecutive steps:

1. Macro-to-micro: Localization of the macroscale deformations to the microscale

2. Resolution of the boundary value problem on the microscale with finite elements 
3. Micro-to-macro: Homogenization of the microscale stresses to compute the macroscopic quantity

4. Resolution of the boundary value problem on the macroscale with finite elements

These steps are repeated for each Gauss point until convergence is obtained on both scales. Despite the method is computationally rather expensive, it is far less than a direct modelling approach.

Kouznetsova et al. (2001) presented the $F E^{2}$ method for the modelling of mechanical problems. In the context of multi-physics and coupling phenomena, the $F E^{2}$ method was first applied to deal with heat conduction problems (Özdemir et al., 2008). Then, it was used for the modelling of saturated porous media (Su et al., 2011; Marinelli, 2013; Van den Eijnden et al., 2016). In the context of CBM recovery, the method has to be extended to partially saturated media. To our knowledge, this method has never been applied to coal before.

With respect to the previous developments, the new model requires the definition of an additional fluid degree of freedom for gas. The presence of gas implies the implementation of the microscale hydraulic constitutive model for partially saturated conditions. In particular, a retention curve is defined for the interface elements, some relative permeabilities curves are introduced to compute the advective fluxes and some equilibrium equations between the phases have to be written. Moreover, the particular case of coal, with sorption properties, requires to compute the gas storage term taking into account the Langmuir's isotherm and a sorption time, and accounts for the swelling/shrinkage due to the sorption/desorption in the solid bodies. This microscale model has been presented and verified by comparison with analytical models in a previous paper (Bertrand et al., 2019). The paper here presents the integration of this model in a multiscale scheme using homogenization techniques whose suitability to manage the transition from one scale to another is well established (Van den Eijnden et al., 2016).

The model is implemented into the numerical code Lagamine developed by the University of Liège (Charlier, 1987). After the presentation of the model, it is used for reservoir modelling. A synthetic case is first considered to demonstrate the ability of the model to obtain production curves similar to those observed for unconventional reservoir. A sensitivity analysis is also performed on the main parameters of the model. Finally, a history matching exercise is conducted.

\section{Hydro-mechanical model}

The double-scale model requires the definition of a Representative Elementary Volume (REV) which is submitted to periodic boundary conditions to enforce the localization of the macroscopic loading. The constitutive laws used to solve the microscale boundary value problem are briefly presented in this section. Finally, microscale quantities are homogenized to solve the macroscale boundary value problem.

\subsection{REV and unit cell}

The REV is built with solid matrix blocks separated by interfaces (cleats). In the case of materials with a specifically built periodic microstructure, one can simply 
choose a periodic unit cell as REV. Unit cells are extensively used for the modelling of human-made materials such as masonry walls (Massart, 2003). It is more questionable for natural materials but coal is most generally described with a periodic matchstick geometry in the literature (Seidle et al., 1992). The multiscale method is however not restricted to periodic materials.

For the sake of simplicity, it is chosen not to use a REV border along a fracture. The kind of REV that may be used with our model is represented in Figure 1. The REV is actually constituted of three parts: the solid matrix, the mechanical interfaces between the grains, and the fluid network (matching the interfaces).

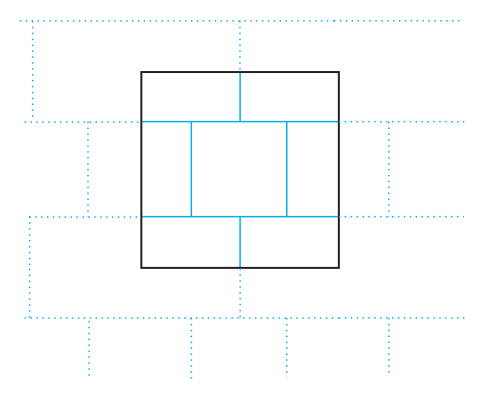

Figure 1: Microstructure with REV borders through the matrix.

Note that it is not strictly necessary to define a REV size from a mechanical point of view. However, a length dimension is necessary to obtain a meaningful permeability tensor. A length factor is therefore introduced to define the REV size. In a practical way, the microscale computations are carried out using a REV with a size of one by one. The macroscopic scale being expressed in meters, the length factor is used for the conversion of all the quantities going to (localization step) or coming from (homogenization steps) the microscale.

\subsection{Decomposition of the microkinematics}

The double-scale modelling approach distinguishes a microscale and a macroscale ${ }^{1}$. Given a point $\hat{\mathcal{P}}$ with a displacement $u_{i}^{M}(\hat{\mathcal{P}})$, the microkinematics $u_{i}^{m}(\hat{\mathcal{P}})$ is identical. For a point $\mathcal{P}$ close to $\hat{\mathcal{P}}$, the displacement can be defined with a Taylor expansion. In the macroscale continuum, higher-order terms of the expansion can be neglected so that the macromechanical displacement field $u_{i}^{M}(\mathcal{P})$ is approximately equal to:

$$
u_{i}^{M}(\mathcal{P}) \approx u_{i}^{M}(\hat{\mathcal{P}})+\frac{\partial u_{i}^{M}(\hat{\mathcal{P}})}{\partial x_{j}}\left(x_{j}-\hat{x}_{j}\right)
$$

where $x_{j}$ and $\hat{x}_{j}$ are respectively the $\mathrm{j}^{\text {th }}$ coordinates of $\mathcal{P}$ and $\hat{\mathcal{P}}$.

\footnotetext{
${ }^{1}$ The superscript $m$ is used to refer to the microscale while the capital letter $M$ denotes macroscopic quantities.
} 
For the microscale, higher-order terms can not be neglected because the continuity is not ensured. The micromechanical displacement field $u_{i}^{m}(\mathcal{P})$ is therefore decomposed in the macromechanical field $u_{i}^{M}(\mathcal{P})$ and a fluctuation displacement field $u_{i}^{f}(\mathcal{P})$ replacing higher order terms:

$$
u_{i}^{m}(\mathcal{P})=u_{i}^{M}(\hat{\mathcal{P}})+\frac{\partial u_{i}^{M}(\hat{\mathcal{P}})}{\partial x_{j}}\left(x_{j}-\hat{x}_{j}\right)+u_{i}^{f}(\hat{\mathcal{P}})
$$

This fluctuation field results of the variations in material properties within the REV. It represents therefore the fine scale deviations with respect to the average fields. As the statement $u_{i}^{m}(\hat{\mathcal{P}})=u_{i}^{M}(\hat{\mathcal{P}})$ has to hold for any point of the macroscale, it follows that

$$
\frac{\partial u_{i}^{M}(\hat{\mathcal{P}})}{\partial x_{j}}\left(x_{j}-\hat{x}_{j}\right)+u_{i}^{f}(\hat{\mathcal{P}}) \ll u_{i}^{M}(\hat{\mathcal{P}})
$$

The validity of the approach is therefore theoretically restricted to situations in which the scale of variation of the macroscopic fields is large compared to the microstructure and its REV. It is the concept of separation of scales (Kouznetsova et al., 2002). Simply written, the microscopic characteristic length $l_{c}$ has to be much smaller than the characteristic fluctuation length $L_{c}$ of the macroscopic deformation fields.

If this assumption holds, the boundary conditions of the microscale boundary value problem (BVP) can be properly dictated by the local macroscale deformation gradient. If the macroscopic field varies considerably in critical regions of high gradient, the assumption of scale separation may not hold. Large spatial gradients are observed, for instance, with strain localization problems (Geers et al., 2010). In this case, a higher-order homogenization technique should be applied (Schröder, 2014). It means the Taylor expansion of the displacement field is not troncated before the second-order term (Kouznetsova et al., 2002). This work is limited to the application of the first-order computational homogenization. It supposes therefore a separation of scales.

In order to develop the hydro-mechanical problem for partially saturated conditions, the water pressure $p_{w}$ and gas pressure $p_{g}$ are also introduced. As for the microscale displacements, the microscale pressure fields of water and gas can be decomposed into macroscale components and microkinematical fluctuations:

$$
p_{w}^{m}(\mathcal{P})=p_{w}^{M}(\hat{\mathcal{P}})+\frac{\partial p_{w}^{M}(\hat{\mathcal{P}})}{\partial x_{j}}\left(x_{j}-\hat{x}_{j}\right)+p_{w}^{f}(\mathcal{P})
$$

and

$$
p_{g}^{m}(\mathcal{P})=p_{g}^{M}(\hat{\mathcal{P}})+\frac{\partial p_{g}^{M}(\hat{\mathcal{P}})}{\partial x_{j}}\left(x_{j}-\hat{x}_{j}\right)+p_{g}^{f}(\mathcal{P})
$$

The requirement of separation of scales implies that

$$
\frac{\partial p_{w}^{M}(\hat{\mathcal{P}})}{\partial x_{j}}\left(x_{j}-\hat{x}_{j}\right)+p_{w}^{f}(\mathcal{P}) \ll p_{w}^{M}(\hat{\mathcal{P}})
$$


and

$$
\frac{\partial p_{g}^{M}(\hat{\mathcal{P}})}{\partial x_{j}}\left(x_{j}-\hat{x}_{j}\right)+p_{g}^{f}(\mathcal{P}) \ll p_{g}^{M}(\hat{\mathcal{P}})
$$

Section 2.4 will present a practical way to verify these requirements.

\subsection{Periodic boundary conditions}

The macroscopic deformation enters the microscale BVP through the boundary conditions (Geers et al., 2010). Dirichlet and Neumann boundary conditions provide an upper and lower bound solution, the former tend to overestimate and the latter to underestimate the equivalent material strength when the REV is not large enough to be fully representative (Suquet, 1987). The periodic boundary conditions, giving a result between these two bounds, are therefore adopted for their efficiency to transfer the macroscale deformation to the microscale REV. This choice has been justified by different authors (Van der Sluis et al., 2000; Terada et al., 2000). Note that the application of periodic boundary conditions does not actually requires a periodic material. For instance, these boundary conditions may be applied for a granular material. However, it is a natural course of action for periodic media such as coal since these boundary conditions enforce the local periodicity of the microscale material behaviour (Van den Eijnden, 2015).

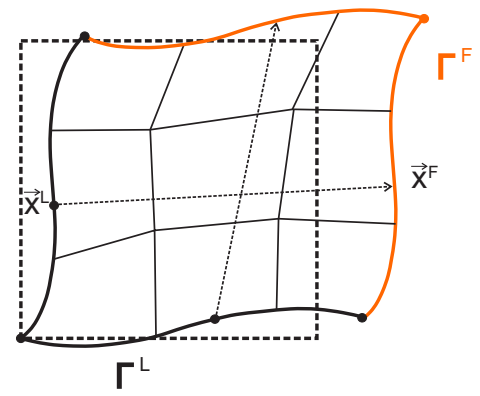

Figure 2: Periodic boundary conditions

The boundary $\Gamma$ enclosing the REV being periodic, it can be subdivided in two parts: the lead part $\Gamma^{L}$ and the follow part $\Gamma^{F}$. The kinematics of any point $x^{F}$ on the follow boundary $\Gamma^{F}$ depends obviously on the kinematics of its homologous $x^{L}$ on the lead boundary $\Gamma^{L}$. The mechanical part of the periodic boundary conditions for the $\mathrm{REV}$ is defined in terms of displacements through the relation

$$
u_{i}^{F}=u_{i}^{L}+\varepsilon_{i j}^{M}\left(x_{j}^{F}-x_{j}^{L}\right)
$$

where $\varepsilon_{i j}^{M}$ is the macroscopic Cauchy strain. A small strain assumption is considered at the microscale.

In the same way, the boundary conditions for water and gas pressures are given by 


$$
p_{w}^{F}=p_{w}^{L}+\frac{\partial p_{w}^{M}}{\partial x_{j}}\left(x_{j}^{F}-x_{j}^{L}\right) \quad \text { (9) } \quad \text { and } \quad p_{g}^{F}=p_{g}^{L}+\frac{\partial p_{g}^{M}}{\partial x_{j}}\left(x_{j}^{F}-x_{j}{ }^{L}\right)
$$

Moreover, the periodic boundary conditions lead to the requirement of the antisymmetry of the boundary traction:

$$
\bar{t}_{i}^{F}+\bar{t}_{i}^{L}=0
$$

And anti-symmetric boundary fluxes:

$$
\bar{q}_{w}^{F}+\bar{q}_{w}^{L}=0
$$

$$
\text { and } \quad \bar{q}_{g}^{F}+\bar{q}_{g}^{L}=0
$$

\subsection{Separation of scales}

The microscopic characteristic length should be much smaller than the characteristic fluctuation length of the macroscopic deformation fields. Applying the macroscopic deformation fields on the boundaries, the microscopic characteristic length is reasonably the length between these boundaries $\left(x_{j}{ }^{F}-x_{j}{ }^{L}\right)$, i.e. the REV size $l_{R E V}$. Then, the macroscopic length depends on the load applied on the REV, i.e. the macroscopic gradient of fluid pressure $\frac{\partial p^{M}}{\partial x_{j}}$ (or written $\nabla p^{M}$ ) for the hydraulic part, and the macroscopic pressure. The macroscopic length is thus defined as the ratio of the pressure over the pressure gradient to verify that

$$
l_{R E V} \ll \frac{p^{M}}{\nabla p^{M}}
$$

to satisfy the separation of scales. It is formulated here for the fluid pressure and pressure gradient (gas or water) since the objective of this work is to model a pressure drop at a production well. It is therefore the most critical quantity.

Moreover, as the size of the REV/unit cell should be very small compared to the macroscale, it is generally consistent to assume the hydraulic flow at the micro level is insensitive to the time variations of the fluid storage at this level. In other words, the microscale problem may be solved under steady-state conditions. It means that boundary conditions dictate the instantaneous pressure changes at the micro level. However, in some cases, it is sometimes necessary to take into account transient effects. For instance, there may be a double porosity effect due to the diffusive flows in the matrix. An instantaneous equilibrium between the pressure and the matrix should only be considered if

$$
\mathcal{T} \ll \frac{p^{M}}{\dot{p}^{M}}
$$

where $\mathcal{T}$ is the characteristic time at the microscale, it is the sorption time in this case. It depends on the diffusion coefficient and the size of the matrix block (Bertrand et al., 2017). The right-hand term refers to the macroscopic characteric time.

If condition (15) is not ensured, a transient computational homogenization could be used to take into account micro-structural inertia effects at the macroscopic level. For instance, (Larsson et al., 2010a,b) avoided the steady state assumption for heat flow at the microscale considering higher order terms in the homogenization procedure. 
An appropriate scale transition has also been presented by (Pham et al., 2013) for the dynamic modelling of a heterogeneous medium subjected to a pulse loading. This transient scheme is not developed in this work as the loading is likely less extreme in the context of reservoir modelling. However, to model more adequately cases where the sorption time is close to the loading characteristic time, it is interesting to introduce the sorption time coefficient in the adsorbed gas pressure evolution computed under pseudo-steady state conditions.

\subsection{Microscale balance equations}

For our applications, the thickness of the coal seam is considered to be very small compared to the depth of the seam, so the influence of the gravity should be very small and is therefore neglected.

Neglecting the body forces, the equilibrium of the REV means the Cauchy stress tensor $\sigma_{i j}^{m}$ has to satisfy ${ }^{2}$ :

$$
\frac{\partial \sigma_{i j}^{m}}{\partial x_{j}}=0
$$

The weak form of the local momentum balance Equation (16) is obtained applying the principle of virtual power considering the admissible virtual velocity field $v_{i}^{* m}$, it reads

$$
\int_{\Omega} \sigma_{i j}^{m} \frac{\partial v_{i}^{* m}}{\partial x_{j}} d \Omega=\int_{\Gamma} \bar{t}_{i} v_{i}^{* m} d \Gamma
$$

where $\Omega$ is the volume of the REV. As the REV is constituted of blocks and interfaces, the boundary $\Gamma$ in Equation (17) can be divided in external boundaries $\Gamma^{e x t}$ (the periodic REV boundaries) and internal boundaries $\Gamma^{\text {int }}$ (the interfaces). The integral over the external boundary is zero given the periodic boundary conditions.

In addition to the mechanical equilibrium, the mass balance equations for water and gas are

$$
\dot{M}_{w}^{m}+\frac{\partial f_{w_{i}}^{m}}{\partial x_{i}}=0 \quad \text { (18) } \quad \text { and } \quad \dot{M}_{g}^{m}+\frac{\partial f_{g_{i}}^{m}}{\partial x_{i}}=0
$$

where $f_{w_{i}}^{m}$ and $f_{g_{i}}^{m}$ are the internal total fluxes of water and gas, and $\dot{M}_{w}^{m}$ and $\dot{M}_{g}^{m}$ represent the variations of the fluid contents. Under the assumption of steady state at the microscale, these latter terms will vanish out. Considering some kinematically admissible virtual fluid pressure fields $p_{w}^{* m}$ and $p_{g}^{* m}$, the fluids mass balance equations may be written in the following weak forms:

$$
\begin{aligned}
& \int_{\Omega}\left[\dot{M}_{w}^{m} p_{w}^{* m}-f_{w_{i}}^{m} \frac{\partial p_{w}^{* m}}{\partial x_{i}}\right] d \Omega=-\int_{\Gamma} \bar{q}_{w}^{m} p_{w}^{* m} d \Gamma \\
& \int_{\Omega}\left[\dot{M}_{g}^{m} p_{g}^{* m}-f_{g_{i}}^{m} \frac{\partial p_{g}^{* m}}{\partial x_{i}}\right] d \Omega=-\int_{\Gamma} \bar{q}_{g}^{m} p_{g}^{* m} d \Gamma
\end{aligned}
$$

\footnotetext{
${ }^{2}$ Small strains are assumed at the microscale. Through this assumption, the Piola-Kirchhoff stress and the Cauchy stress tensors can be assumed approximately equal.
} 


\subsection{Constitutive laws}

The constitutive microscale model is constituted of some mechanical laws for the matrix and the fracture, the fluid density variation law and the multiphase flow model. The multiscale framework presented in this section is not restricted to some particular constitutive laws. Based on the developments presented in (Bertrand et al., 2019), the laws used for the coalbed modelling are shortly presented hereafter. It was chosen to implement the microscale mechanical constitutive laws of the multiscale approach with increments from the initial state given that elastic mechanical laws are used.

\subsubsection{Matrix}

Constitutive laws are defined for the mechanical behaviour of the matrix and for the adsorbed gas pressure evolution.

\section{Solid constitutive law.}

It is assumed the sophistication of the material behaviour is obtained from the assembly of different constituents. It is thus not unreasonable to consider an isotropic linear elastic relation for the matrix. Considering sorption-induced strain by analogy with thermal expansion/contraction, the elastic law writes

$$
\Delta \sigma_{i j}=2 G_{m} \Delta \varepsilon_{i j}+\lambda_{m} \Delta \bar{\varepsilon} \delta_{i j}+K_{m} \Delta \varepsilon_{v_{s}} \delta_{i j}
$$

where $\varepsilon_{i j}$ is the small-strain tensor given by $\frac{1}{2}\left(\frac{\partial u_{i}}{\partial x_{j}}+\frac{\partial u_{j}}{\partial x_{i}}\right), \bar{\varepsilon}=\varepsilon_{i j} \delta_{i j}$ with $\delta_{i j}$ the Kronecker's symbol, $G_{m}$ is the Coulomb's modulus and $\lambda_{m}$ is the first Lamé parameter, $K_{m}=\lambda_{m}+\frac{2 G_{m}}{3}$ is the bulk modulus of the matrix and $\Delta \varepsilon_{v_{s}}$ is the volumetric sorption-induced strain. This sorption-induced strain depends on the adsorbed gas content $V_{g}^{A d}\left[\mathrm{~m}^{3} / \mathrm{kg}\right]$ and a linear coefficient $\beta_{\varepsilon}\left[\mathrm{kg} / \mathrm{m}^{3}\right]$ (Cui and Bustin, 2005):

$$
\Delta \varepsilon_{v_{s}}=\beta_{\varepsilon} \Delta V_{g}^{A d}=\beta_{\varepsilon} \frac{M_{m_{g}}}{R T} \frac{1}{\rho_{g, s t d} \rho_{c}} \Delta p_{g}^{A d}
$$

where $R$ is the universal gas constant $(8.3143 \mathrm{~J} / \mathrm{mol} \cdot K), T[K]$ is the absolute temperature, $M_{m_{g}}$ is the molecular mass of the gas $(0.016 \mathrm{~kg} / \mathrm{mol}$ for methane and $0.044 \mathrm{~kg} / \mathrm{mol}$ for carbon dioxide), $\rho_{g, s t d}$ is the gas density at standard conditions, $\rho_{c}$ is the coal density, and $p_{g}^{A d}$ is the adsorbed gas pressure in the matrix.

In addition, the reservoir conditions require the presence of initial stresses $\sigma_{i j_{0}}$. This stress is associated to the reference state for which the strain is zero. Then, the stress tensor at a given time is

$$
\sigma_{i j}=\sigma_{i j_{0}}+\Delta \sigma_{i j}
$$

where $\Delta \sigma_{i j}$ represents the increment of stress between the initial and current stresses. 
Adsorbed gas pressure evolution.

At equilibrium, the adsorbed gas pseudo-pressure is given by

$$
p_{g}^{A d}=\frac{R T}{M_{m_{g}}} \rho_{g, s t d} \rho_{c} \frac{V_{L} \cdot p}{P_{L}+p}
$$

where $V_{L}$ and $P_{L}$ are the Langmuir's parameters (Langmuir, 1918), and $p$ depends on the macroscopic fluid pressures (which is consistent with the separation of scales):

$$
p=\max \left(p_{w}^{M}, p_{g}^{M}\right)
$$

If the hypothesis of an instantaneous equilibrium between the pressure in the cleats and the adsorbed gas content in the matrix is too restrictive, it is interesting to introduce a sorption time $\mathcal{T}$ to take into account the delay imposed by the diffusion process into the matrix. The average adsorbed gas pressure $p_{g}^{A d}$ is then given by

$$
p_{g}^{A d}(t)=p_{g}^{A d}(t-\Delta t)+\frac{1}{\mathcal{T}}\left(p_{g}^{A d, l i m}-p_{g}^{A d}(t-\Delta t)\right) \Delta t
$$

with $p_{g}^{A d, \text { lim }}$ evaluated with Equation (25). The value of the adsorbed gas pressure $p_{g}^{A d}$ at the end of the time step is more accurately determined following a sub-stepping procedure by replacing $\Delta t$ with $\delta t$.

\subsubsection{Cleats}

Concerning the cleats, a mechanical law and a channel flow model are defined.

\section{Interface mechanical law.}

The mechanical law for the interfaces is defined by two parameters, the normal stiffness $K_{n}$ and tangential stiffness $K_{s}$, which define the relation between the stresses and the fracture displacements. The tangential stiffness is considered constant, $K_{s}=$ $K_{s}^{0}$, while a Bandis-type law is used for the normal stiffness (Bandis et al., 1983):

$$
K_{n}=\frac{K_{n}^{0}}{\left(1+\frac{h-h_{0}}{h_{0}}\right)^{2}}
$$

where $K_{n}^{0}$ is the stiffness corresponding to the initial aperture $h_{0}$. This initial aperture is actually defined for a null stress. Thus, when considering some initial stresses, the initial normal closure of a given fracture is given by

$$
\Delta h_{0}=\frac{-\sigma_{0}^{\prime} h_{0}}{K_{n}^{0} h_{0}+\sigma_{0}^{\prime}}
$$

where $\sigma_{0}^{\prime}$ is the effective stress normal to the fracture wall. In this way, the cleat aperture may evolve with the variation in fluid pressure without the total stress changing.

This effective stress is the total stress reduced by the fluid pressures weighted by 
the degree of saturation of each phase:

$$
\sigma_{i j}^{\prime}=\sigma_{i j}-\left[S_{r} p_{w}^{M}+\left(1-S_{r}\right) p_{g}^{M}\right] \delta_{i j}
$$

where $\delta_{i j}$ is the Kronecker symbol. Note that this definition of the effective stress in the fracture does not require a Biot's coefficient as usually used in macroscale constitutive laws for homogenized medium. The saturation degree $S_{r}\left(p_{c}\right)$ is obtained from the retention curve:

$$
p_{c}=p_{w}^{M}-p_{g}^{M}=p_{e} \cdot\left(S_{r}^{*}\right)^{-\frac{1}{\lambda}}
$$

where $p_{e}$ is the entry capillary pressure and $\lambda$ is an index representing the size distribution of the capillary tubes. $S_{r}^{*}$ is a normalized saturation:

$$
S_{r}^{*}=\frac{S_{r}-S_{r, \text { res }}}{1-S_{r, \text { res }}-S_{r_{g}, \text { res }}}
$$

with $S_{r, \text { res }}$ and $S_{r_{g} \text {,res }}$ the residual water and gas saturations.

Since the tangential stiffness is constant, the initial tangential closure is directly:

$$
\Delta s_{0}=\frac{\tau_{0}}{K_{s}^{0}}
$$

where $\tau_{0}$ is the initial tangential stress. Given the initial stresses $\sigma_{x x_{0}}$ and $\sigma_{y y_{0}}$, and the orientation $\theta$ of the fracture with the horizontal, the normal and tangential stresses are computed as

$$
\begin{gathered}
\sigma_{0}^{\prime}=\sigma_{x x_{0}}^{\prime} \cos ^{2}(\theta)+\sigma_{y y_{0}}^{\prime} \sin ^{2}(\theta) \\
\tau_{0}=\frac{1}{2}\left(\sigma_{y y_{0}}-\sigma_{x x_{0}}\right) \sin (2 \theta)
\end{gathered}
$$

Finally, the relations between stresses and displacements are:

$$
\begin{gathered}
\sigma_{0}^{\prime}+\Delta \sigma^{\prime}=\frac{K_{n}^{0} h_{0}}{h}\left(\Delta h_{0}+\Delta h\right) \\
\tau_{0}+\Delta \tau=K_{s}^{0}\left(\Delta s_{0}+\Delta s\right)
\end{gathered}
$$

\section{Channel flow model.}

From the constitutive microscale model, the interface mass flux of water is given by

$$
\omega_{w}=-\rho_{w} h_{b} k_{r w} \frac{h_{b}^{2}}{12} \frac{1}{\mu_{w}} \frac{d p_{w}^{m}}{d s}=-\rho_{w} \frac{k_{r w}}{\mu_{w}} \kappa(s) \frac{d p_{w}^{m}}{d s}
$$

where $\kappa(s)$ is the geometric transmissivity function along the channel with $s$ the coordinate along this channel. This equation provides the relation between the water pressure gradient along the channel and the channel water mass flux. The steady state flow condition at the microscale implies that the mass flux $\omega_{w}$ is constant over the length of the channel. Moreover, considering the scale separability, the fluid pressure variation is very small compared to the REV size and has a negligible effect on the 
fluid density $\rho_{w}$. The density is thus considered to be constant throughout the REV. Under isothermal conditions, the water density is only dependent on the macroscale water pressure. In the same way, the relative permeability $k_{r w}$ is only dependent on the macroscale fluid pressures. As presented in the paper on the cleat-scale model (Bertrand et al., 2019), the relative permeability curves which are used are

$$
k_{r w}=\frac{S_{r}^{2}}{2}\left(3-S_{r}\right) \quad(39) \quad k_{r g}=\left(1-S_{r}\right)^{3}
$$

The density $\rho_{w}$ of the liquid phase evolves with the macroscale pressure $p_{w}^{M}$ according to:

$$
\rho_{w}=\rho_{w_{0}}\left(1+\frac{p_{w}^{M}-p_{w_{0}}^{M}}{\chi_{w}}\right)
$$

where $\rho_{w_{0}}$ is the liquid density at the pressure $p_{w_{0}}^{M}$ and $1 / \chi_{w}$ is the liquid compressibility. At $20^{\circ} \mathrm{C}, 1 / \chi_{w}=5 \cdot 10^{-10} \mathrm{~Pa}^{-1}$.

Then, we can write the mass fluxes over the channel between $s_{1}$ and $s_{2}$ as

$$
\begin{aligned}
\omega_{w}^{l} & =\left[p_{w}^{m}\left(s_{2}\right)-p_{w}^{m}\left(s_{1}\right)\right] \frac{\rho_{w}\left(p_{w}^{M}\right) k_{r w}\left(p_{w}^{M}, p_{g, f}^{M}\right)}{\mu_{w}}\left(\int_{s_{1}}^{s_{2}} \frac{1}{\kappa(s)} d s\right)^{-1} \\
& =\left[p_{w}^{m}\left(s_{2}\right)-p_{w}^{m}\left(s_{1}\right)\right] \frac{\rho_{w}\left(p_{w}^{M}\right) k_{r w}\left(p_{w}^{M}, p_{g, f}^{M}\right)}{\mu_{w}} \Phi^{l}
\end{aligned}
$$

where $\Phi^{l}$ is the geometric transmissivity of the channel.

For gas, the interface mass flux is:

$$
\begin{aligned}
\omega_{g} & =-\rho_{g} h_{b} k_{r g} \frac{h_{b}^{2}}{12} \frac{1}{\mu_{g}} \frac{d p_{g}^{m}}{d s}-H_{g} \rho_{g} h_{b} k_{r w} \frac{h_{b}^{2}}{12} \frac{1}{\mu_{w}} \frac{d p_{w}^{m}}{d s} \\
& =-\frac{\rho_{g} k_{r g}}{\mu_{g}} \kappa \frac{d p_{g}^{m}}{d s}-H_{g} \frac{\rho_{g} k_{r w}}{\mu_{w}} \kappa \frac{d p_{w}^{m}}{d s}
\end{aligned}
$$

where the first term describes the advection of gas and the second term is the dissolved gas in the advective water flow. Henry's coefficient $H_{g}$ allows determining the dissolved gas volume in the water.

The classical ideal gas equation of state is used to write the gas density in the fractures as

$$
\rho_{g}=\frac{M_{m_{g}}}{R T} p_{g}^{M}
$$

where $p_{g}^{M}$ is the macroscale gas pressure.

Note that given the negligible effect of the variations of the fluid pressures in the REV on the fluid densities (due to scale separability), diffusive flows are not considered in the fractures. Over the channel, the gas mass flux is then evaluated by:

$$
\omega_{g}^{l}=\left[p_{g}^{m}\left(s_{2}\right)-p_{g}^{m}\left(s_{1}\right)\right] \frac{\rho_{g} k_{r g}}{\mu_{g}} \Phi^{l}+\left[p_{w}^{m}\left(s_{2}\right)-p_{w}^{m}\left(s_{1}\right)\right] H_{g} \rho_{g} \frac{k_{r w}}{\mu_{w}} \Phi^{l}
$$


In this work, no flow is directly considered in the matrix ${ }^{3}$. The fluxes $\bar{q}_{w}$ and $\bar{q}_{g}$ appearing in the balance equations and boundary conditions are therefore given by the fluxes in the interfaces, $\omega_{w}^{l}$ and $\omega_{g}^{l}$.

\subsection{Microscale numerical solution}

The microscale problem is solved iteratively in two parts, starting with the mechanical problem and then finding the different flows for the given fracture apertures. The sequence is repeated until convergence.

\subsubsection{Mechanical part}

The system of field Equation (17) governing the microscale BVP is solved numerically using a full Newton-Raphson scheme. Knowing the configuration at time $t$ in equilibrium with the boundary conditions, the objective is to find the configuration in equilibrium at the end of the time step. An updated configuration is first guessed, for which it is likely the equilibrium is not met. Residuals therefore appear in the balance equations and the aim is to find a new configuration for which the residuals vanish. In this purpose, the volume is discretized with finite elements.

For the matrix, a 4-node element with 4 integration points is adopted for the spatial discretization. For the discretization of the cleats, one-dimensional elements with 2 integration points are used.

Assembling the element stiffness matrices and the element nodal force vectors of the continuum and the interfaces, the global stiffness matrix $\left[K_{m m}\right]$ yields the incremental relation between the nodal displacement $\{u\}$ and the nodal force $\{f\}$ :

$$
\left[K_{m m}\right]\{\delta u\}=\{\delta f\}
$$

This relation is valid for constant fluid pressures. The hydraulic part of the problem is now solved separately.

\subsubsection{Hydraulic part}

The explicit description of the fluid network is solved separately from the mechanical problem in order to find the profiles of the gas and water pressures that respect the boundary conditions and, at the same time, have some average values of the pressure fields equal to the macroscopic water and gas pressures.

The mass conservation along the channel leads to write that, for each node of the hydraulic network, the sum of the input flows must be equal the sum of output flows:

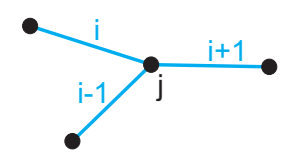

$$
\omega^{i-1}+\omega^{i}+\omega^{i+1}=0
$$

with $\omega^{i} \propto \Phi^{i}\left(p_{j}-p_{j-1}\right)$

\footnotetext{
${ }^{3}$ Diffusive flows in the matrix are indirectly considered using a sorption time coefficient to compute the mean adsorbed gas pressure evolution with Equation (27).
} 
The hydraulic system to solve is written considering all the connections between the nodes. For instance, Figure 3 gives a basic example of a channel network.

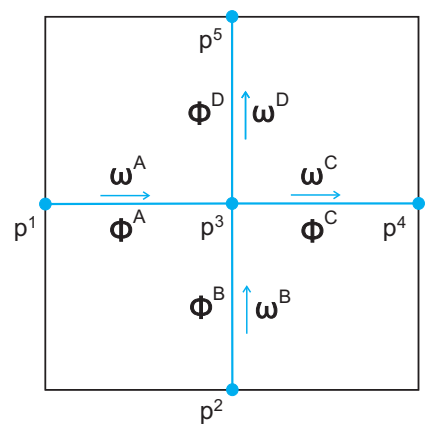

Figure 3: Example of a channel network.

For this configuration, taking into account the homologous connectivity of lead and follow node couples over the periodic boundaries and the conditions given by the macroscopic pressure gradient (Equation 9), and applying the macroscopic pressure at one of the nodes of the hydraulic network, the well-posed system to solve is

$$
\frac{\rho_{w} k_{r w}}{\mu_{w}}\left[\begin{array}{ccccc}
\Phi^{A} & 0 & -\Phi^{A}-\Phi^{C} & \Phi^{C} & 0 \\
0 & 1 & 0 & 0 & 0 \\
-\Phi^{A} & -\Phi^{B} & \Phi^{A}+\Phi^{B}+\Phi^{C}+\Phi^{D} & -\Phi^{C} & -\Phi^{D} \\
-1 & 0 & 0 & 1 & 0 \\
0 & -1 & 0 & 0 & 1
\end{array}\right]\left(\begin{array}{c}
p_{w}^{1} \\
p_{w}^{2} \\
p_{w}^{3} \\
p_{w}^{4} \\
p_{w}^{5}
\end{array}\right)=\left(\begin{array}{c}
0 \\
p_{w}^{M} \\
0 \\
\left(\Delta p_{w}\right)_{x}^{M} \\
\left(\Delta p_{w}\right)_{y}^{M}
\end{array}\right)
$$

For gas, as gas dissolved in the water may be considered in the cleats, the advective flows of water implies some gas mass fluxes. Actually, since densities, relative permeabilities and saturation are only determined based on the macroscopic fluid pressures, the microscopic water pressures are already known before solving the system for gas. Term relative to dissolved gas may be therefore written as an independent term of the 
equation:

$$
\begin{array}{r}
\frac{\rho_{g} k_{r g}}{\mu_{g}}\left[\begin{array}{ccccc}
\Phi^{A} & 0 & -\Phi^{A}-\Phi^{C} & \Phi^{C} & 0 \\
0 & 1 & 0 & 0 & 0 \\
-\Phi^{A} & -\Phi^{B} & \Phi^{A}+\Phi^{B}+\Phi^{C}+\Phi^{D} & -\Phi^{C} & -\Phi^{D} \\
-1 & 0 & 0 & 1 & 0 \\
0 & -1 & 0 & 0 & 1
\end{array}\right]\left(\begin{array}{l}
p_{g}^{1} \\
p_{g}^{2} \\
p_{g}^{3} \\
p_{g}^{4} \\
p_{g}^{5}
\end{array}\right) \\
=-\frac{\rho_{g}^{d} k_{r w}}{\mu_{w}}\left[\begin{array}{ccccc}
\Phi^{A} & 0 & -\Phi^{A}-\Phi^{C} & \Phi^{C} & 0 \\
0 & \Phi^{B} & -\Phi^{B}-\Phi^{D} & 0 & \Phi^{D} \\
-\Phi^{A} & -\Phi^{B} & \Phi^{A}+\Phi^{B}+\Phi^{C}+\Phi^{D} & -\Phi^{C} & -\Phi^{D}
\end{array}\right]\left(\begin{array}{c}
p_{w}^{1} \\
p_{w}^{2} \\
p_{w}^{3} \\
p_{w}^{4} \\
p_{w}^{5}
\end{array}\right)+\left(\begin{array}{c}
p_{g}^{M} \\
0 \\
\left(\Delta p_{g}\right)_{x}^{M} \\
\left(\Delta p_{g}\right)_{y}^{M}
\end{array}\right)
\end{array}
$$

\subsection{Homogenized macroscale response}

Once the equilibrium is obtained, the micro-to-macro transition is derived from the Hill-Mandel macro-homogeneity condition which requires the average microscale work to be equal to the macroscale work (Hill, 1965). The macroscale stresses, fluxes and fluid masses are obtained by homogenization. The macroscale stiffness matrix is presented in this section as well.

\subsubsection{Stresses}

The macroscopic stresses are obtained from the REV by volume averaging:

$$
\sigma_{i j}^{M}=\frac{1}{\Omega} \int_{\Omega} \sigma_{i j}^{m} d \Omega
$$

\subsubsection{Fluid fluxes}

The macroscale fluxes are the integrals of the microscale boundary fluxes:

$$
\begin{aligned}
f_{w_{i}}^{M} & =\frac{1}{\Omega} \int_{\Gamma} \bar{q}_{w}^{m} x_{i} d \Gamma \\
f_{g_{i}}^{M} & =\frac{1}{\Omega} \int_{\Gamma} \bar{q}_{g}^{m} x_{i} d \Gamma
\end{aligned}
$$

Under steady-state conditions at the micro-level, the macroscale fluxes are therefore the sum of the fluxes on the nodes belonging to the follow boundary. The direction of the microscopic flow depending on the orientation of the cleats is explicitly taken into account when solving the hydraulic system. At the end, the homogenized horizontal flow is determined from the flows on the vertical border of the REV and the vertical homogenized flow is known from the flows on the horizontal border. 


\subsubsection{Fluid masses}

The macroscopic fluid contents $M_{w}^{M}$ and $M_{g}^{M}$ are directly defined as the total amounts of fluid in the REV. As water is assumed only in the fractures, the water content $M_{w}^{M}$ is given by ( $\rho_{w}$ is constant over the volume):

$$
M_{w}^{M}=\frac{1}{\Omega} \int_{\Omega_{w}^{i n t}} \rho_{w} d \Omega=\rho_{w} S_{r} \phi_{f}
$$

with $\rho_{w}$ constant over the REV and where $S_{r}$ is defined as $\frac{\Omega_{w}^{\text {int }}}{\Omega^{\text {int }}}$ and $\phi_{f}$ as $\frac{\Omega^{\text {int }}}{\Omega}$.

The gas content $M_{g}^{M}$ is the amount of gas in the interface (gas phase and dissolved gas in the water) and the adsorbed gas in the matrix for coal.

$$
\begin{aligned}
M_{g}^{M} & =M_{g, f}^{g}+M_{g, f}^{d}+M_{g, m}^{A d} \\
& =\frac{1}{\Omega}\left(\int_{\Omega_{g}^{i n t}} \rho_{g} d \Omega+\int_{\Omega_{w}^{i n t}} \rho_{g}^{d} d \Omega+\int_{\Omega} \rho_{g}^{A d} d \Omega\right) \\
& =\rho_{g}\left(1-S_{r}\right) \phi_{f}+\rho_{g}^{d} S_{r} \phi_{f}+\rho_{g}^{A d} \cdot\left(1-\phi_{f}\right)
\end{aligned}
$$

with $\rho_{g}$ constant over the REV. Note $\rho_{g}^{A d}$ is directly related to the adsorbed gas pressure $p_{g}^{A d}$. Thus, considering the evolution of $p_{g}^{A d}$ taking into account the sorption time (Equation 27), a pseudo-steady state is actually considered for the hydraulic problem. The fluid mass storage terms $\dot{M}_{w}^{M, t}$ and $\dot{M}_{g}^{M, t}$ are obtained with some finite difference approximations over the time interval $\Delta t$ :

$$
\begin{gathered}
\dot{M}_{w}^{M, t} \approx \frac{M_{w}^{M, t}-M_{w}^{M, t-\Delta t}}{\Delta t} \\
\dot{M}_{g}^{M, t} \approx \frac{M_{g}^{M, t}-M_{g}^{M, t-\Delta t}}{\Delta t}
\end{gathered}
$$

\subsubsection{Macroscale stiffness matrix}

The macroscale computations are governed by:

$$
\left[\begin{array}{lll}
{\left[K_{m m}^{M}\right]_{(4 x 4)}} & {\left[K_{m w}^{M}\right]_{(4 x 3)}} & {\left[K_{m g}^{M}\right]_{(4 x 3)}} \\
{\left[K_{w m}^{M}\right]_{(3 x 4)}} & {\left[K_{w w}^{M}\right]_{(3 x 3)}} & {\left[K_{w g}^{M}\right]_{(3 x 3)}} \\
{\left[K_{g m}^{M}\right]_{(3 x 4)}} & {\left[K_{g w}^{M}\right]_{(3 \times 3)}} & {\left[K_{g g}^{M}\right]_{(3 x 3)}}
\end{array}\right]\left\{\begin{array}{c}
\left\{\delta \varepsilon^{M}\right\}_{(4)} \\
\left\{\begin{array}{c}
\delta p_{w}^{M} \\
\delta p_{w}^{M}
\end{array}\right\}_{(3)} \\
\left\{\begin{array}{c}
\delta p_{g}^{M} \\
\delta p_{g}^{M}
\end{array}\right\}_{(3)}
\end{array}\right\}=\left\{\begin{array}{l}
\left\{\delta \sigma^{M}\right\}_{(4)} \\
\left\{\begin{array}{c}
\delta f_{w}^{M} \\
\delta \dot{M}_{w}^{M}
\end{array}\right\}_{(3)} \\
\left\{\begin{array}{c}
\delta f_{g}^{M} \\
\delta \dot{M}_{g}^{M}
\end{array}\right\}_{(3)}
\end{array}\right\}
$$

which can be summarized as

$$
\left[A^{M}\right]_{(10 \times 10)}\left\{\delta U^{M}\right\}_{(10)}=\left\{\delta \Sigma^{M}\right\}_{(10)}
$$


where $\left[A^{M}\right]$ is the macroscale stiffness matrix, $\left\{\delta U^{M}\right\}$ contains the infinitesimal variations of the macroscale variables and $\left\{\delta \Sigma^{M}\right\}$ is their responses. This stiffness matrix $\left[A^{M}\right]$ is obtained by numerical perturbations.

\section{Coalbed methane production modelling}

In this section, the multiscale model is applied to the modelling of coalbed methane production from a wellbore. A synthetic production scenario is first considered to perform a sensitivity analysis. Finally, a real production scenario is considered with the Horseshoe Canyon case.

\subsection{Reference case}

The macroscale mesh and the geometry of the microstructure considered for the reference simulation is given in Figure 4.

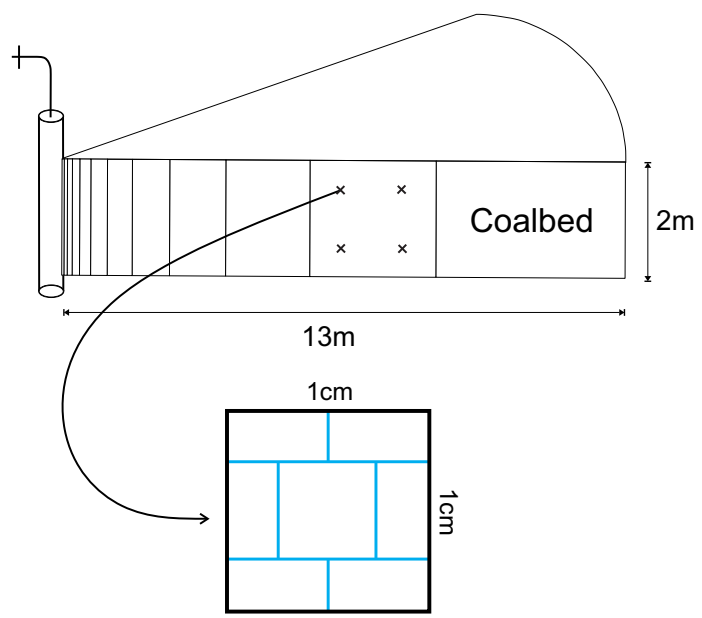

Figure 4: Macroscale mesh and REV geometry.

REV is made up few blocks arranged in staggered rows with continuous horizontal cleats. The size of the unit cell is $1 \mathrm{~cm}$ over $1 \mathrm{~cm}$ allowing to model very few cleats to correspond to typical cleats spacings. All the parameters defining the reference case are reported in Table 1 . The seam is $2 m$ high and the vertical displacement is blocked on the bottom boundary while a $5 \mathrm{MPa}$ overburden pressure is applied on the top boundary. Axisymmetric conditions are considered around the well. The smallest element close to the well is $5 \mathrm{~cm}$ wide. The size of the elements increases with a geometric factor of 1.5 , it means 12 elements represent $13 \mathrm{~m}$. The drained volume is approximately $1000 \mathrm{~m}^{3}$ (external hydraulic borders are impermeable). An actual reservoir is certainly larger but this synthetic reservoir allows us to test very quickly dozens of parameter sets since there are few macroscopic elements (less computation time) and the time to drain the reservoir is not too long to achieve the bell shape of the production curve (less time to simulate). A larger reservoir is modelled later with the Horseshoe Canyon 
case. The drainage radius certainly influences the time-scale of the production but not the observation we can make of the influence that the different parameters have on the production curve. Unfortunately, it is not straightforward to represent the simulation with dimensionless axis since many physical phenomena are involved.

Water and gas pressures are initialized to $3 M P a$, the reservoir is water saturated. Given the overburden pressure of $5 M P a$, the effective stress in the cleats is $2 M P a$. Note the cleat aperture $20 \mu \mathrm{m}$ in Table 1 is given for a null stress, as encoded by the user in the Lagamine data file. At the initial effective stress state, the cleat aperture is actually $10 \mu \mathrm{m}$ and the normal stiffness $225 \cdot 10^{9} \mathrm{~Pa} / \mathrm{m}$.

\begin{tabular}{lr}
\hline Parameters & Values \\
\hline & \\
Seam thickness $(m)$ & 2 \\
Drainage radius $(m)$ & 13 \\
Temperature $(\mathrm{K})$ & 293.15 \\
Water pressure $(\mathrm{Pa})$ & $3 \mathrm{E} 6$ \\
Gas pressure $(\mathrm{Pa})$ & $3 \mathrm{E} 6$ \\
Overburden pressure $(\mathrm{Pa})$ & $5 \mathrm{E} 6$ \\
Well transmissibility $\mathrm{T}\left(\mathrm{m}^{3}\right)$ & $1 \mathrm{E}-10$ \\
Coal density $\rho_{c}\left(\mathrm{~kg} / \mathrm{m}^{3}\right)$ & 1500 \\
Matrix Young's modulus $E_{m}(\mathrm{~Pa})$ & $1.21 \mathrm{E} 9$ \\
Matrix Poisson's ratio $\mathrm{V}_{m}$ & 0.16 \\
Matrix width $w(\mathrm{~m})$ & -0.01 \\
Cleat aperture ${ }^{*} \mathrm{~h}(\mathrm{~m})$ & $20 \mathrm{E}-6$ \\
Cleat normal stiffness ${ }^{*} K_{n}(\mathrm{~Pa} / \mathrm{m})$ & $100 \mathrm{E} 9$ \\
Cleat shear stiffness $K_{s}(\mathrm{~Pa} / \mathrm{m})$ & $100 \mathrm{E} 9$ \\
Minimum cleat aperture $(\mathrm{m})$ & $1 \mathrm{E}-6$ \\
Sorption time $\mathcal{T}$ (hours) & 10 \\
Langmuir volume $V_{L}\left(\mathrm{~m}^{3} / \mathrm{kg}\right)$ & 0.02 \\
Langmuir pressure $P_{L}(\mathrm{~Pa})$ & $1.5 \mathrm{E} 6$ \\
Matrix shrinkage coefficient $\beta_{\varepsilon}\left(\mathrm{kg} / \mathrm{m}^{3}\right)$ & 0.4 \\
Entry capillary pressure $\mathrm{p}_{e}(\mathrm{~Pa})$ & $1 \mathrm{E} 5$ \\
Distribution index $\lambda$ & 0.25 \\
Residual water saturation $S_{r, r e s}$ & 0.1 \\
Residual gas saturation & 0.0 \\
& \\
\hline &
\end{tabular}

Table 1: Parameters defining the reference case. ${ }^{*}$ Aperture and stiffness given for a null stress.

The modelling consists in simulating a drop in the pressure at the well from $3 \mathrm{MPa}$ to $1 \mathrm{MPa}$ in one day. This pressure drawdown causes the water and gas to flow towards the well. Figure 5 presents the water pressure profile obtained due to the pumping. 


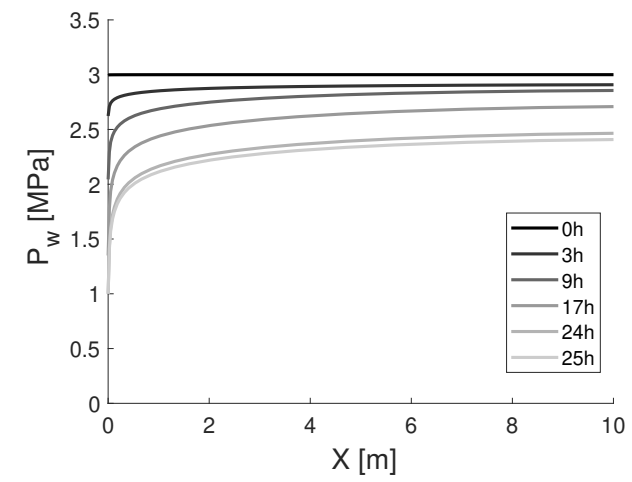

Figure 5: Reference case parameters: water pressure drawdown profile for different times.

The most critical water pressure gradient is observed close to the well at the exact time we stop decreasing the well pressure to maintain it at a constant value of $1 M P a$, i.e. at $24 \mathrm{~h}$ in this case. At this time, the $0.05 \mathrm{~m}$ wide element next to the well shows a water pressure difference of $0.44 \mathrm{MPa}$ between its left edge $\left(p_{\text {well }}=1 \mathrm{MPa}\right)$ and its center $\left(p_{\text {cent }}=1.44 \mathrm{MPa}\right)$. It corresponds to a pressure gradient of $17.6 \mathrm{MPa} / \mathrm{m}$. This results obviously depends on the permeability of the reservoir, it is about $10^{-14} \mathrm{~m}^{2}(10 \mathrm{mD})$ for this simulation. Here, the water pressure profile gives a critical macroscopic characteristic length of $6.9 \mathrm{~cm}$ over the first half of the first element.

$$
L_{c_{\text {crit }}}=\bar{p} \cdot \frac{\Delta x}{\Delta p}=\frac{p_{\text {cent }}+p_{\text {well }}}{2} \frac{\Delta x}{p_{\text {cent }}-p_{\text {well }}}=0.069 \mathrm{~m}
$$

Over the second half of this element, the characteristic length is already $22.6 \mathrm{~cm}$, largely greater than the REV size of $1 \mathrm{~cm}$. For comparison, the water pressure gradient in the second half of the $12^{\text {th }}$ element (most external element in the mesh considered) is only $1.9 \mathrm{kPa} / \mathrm{m}$ at the same time. In this area, the separation between the scales is more than 6 orders of magnitude.

For the same pressure drop of $2 \mathrm{MPa}$, different times of loading are now tested leading to different pressure rates at the well. Figure 6 shows the evolution of the critical length with the water pressure drop rate. 


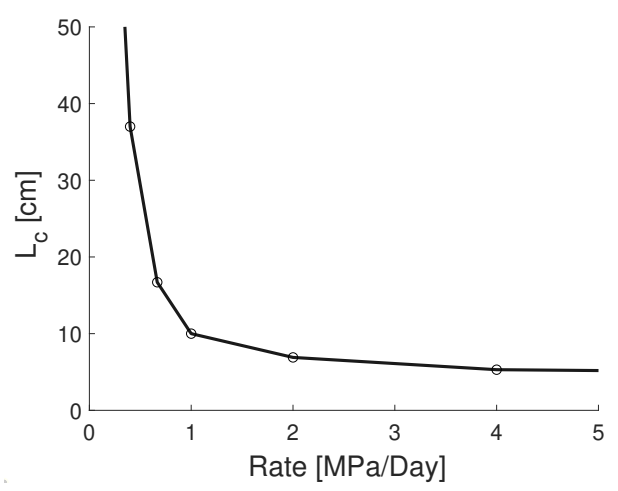

Figure 6: Reference case parameters: evolution of the critical characteristic length with the loading rate.

The lower the rate, the larger the characteristic length, the greater the separation between the scales. The minimum characteristic length will be obtained for a pulse loading, this value can be a priori determined for the given macroscale mesh. Indeed, the pressure at the well is instantaneously set to the final pressure of $1 \mathrm{MPa}$ while the pressure at the center of the first element is still the initial pressure of $3 \mathrm{MPa}$. In this particular case, the mean pressure is equal to the difference of pressure between the well and the center of the first element. Then, the minimum critical characteristic length is directly the length of the half of the first element, i.e. $2.5 \mathrm{~cm}$, which is still greater than the size of the REV. Considering an even more critical case where the well pressure is dropped instantaneously to a very small value compared to its initial value ( $p_{\text {well }} \ll p_{\text {cent }}$ ), then the macroscale characteristic length tends to half of the length of first half-element, $\frac{5 \mathrm{~cm}}{4}>1 \mathrm{~cm}$. The macroscale mesh has therefore to be chosen with care so that its smallest element is large enough to still ensure the separation of scales and small enough to avoid numerical oscillations (depending also on the permeability and the hydraulic storage). From the discussion above, taking a priori the smallest element of the macroscale mesh with a size at least larger than four times the size of the REV ensures the macroscale is larger than the microscale for any pressure drop at any time in any area. Depending how far is the loading compared to a pulse (depending on the permeability), the macroscopic characteristic length will be very larger than the microscopic characteristic length. This can be verified a posteriori.

In the early stages, the permeability to gas is lower than the one to water as the reservoir is initially saturated. For the same loading at the well, the pressure gradient in the first element is therefore higher for gas than water. A critical gradient of $39.8 \mathrm{MPa} / \mathrm{m}$ is obtained in the first half of the first element for the well pressure drop of $2 \mathrm{MPa}$ in one day, it corresponds to a macroscopic characteristic length of $4.0 \mathrm{~cm}$. The minimal characteristic length is the same as water.

From a time point of view, the rate of pressure drop is $2 \mathrm{MPa} /$ day at the well. After one day, at the time the well pressure reaches its minimum of $1 M P a$, the macroscopic characteristic time is thus 0.5 day (Equation 15). This characteristic time is therefore lower than the sorption time $\mathcal{T}$ set to 1 day which is used in Equation (27) to account for diffusion in the coal matrix. As a double porosity effect is not negligible at the most 
critical time close to the well for a concevable sorption time, it was therefore desirable to take into account the sorption time to compute the adsorbed gas pressure evolution in the matrix.

Water and gas production rates are plotted in Figure 7 for one year and half. Water production peaks after one day, at less than $2 \mathrm{~m}^{3} /$ day. Then, desaturation of the reservoir increases the gas permeability and gas production peaks few days after the water, at more than $400 \mathrm{~m}^{3} /$ day (standard conditions). The bell shape of the production curve is related to the size of the reservoir and the outer boundary conditions. With a small reservoir and an impermeable boundary, a plateau is not observed.

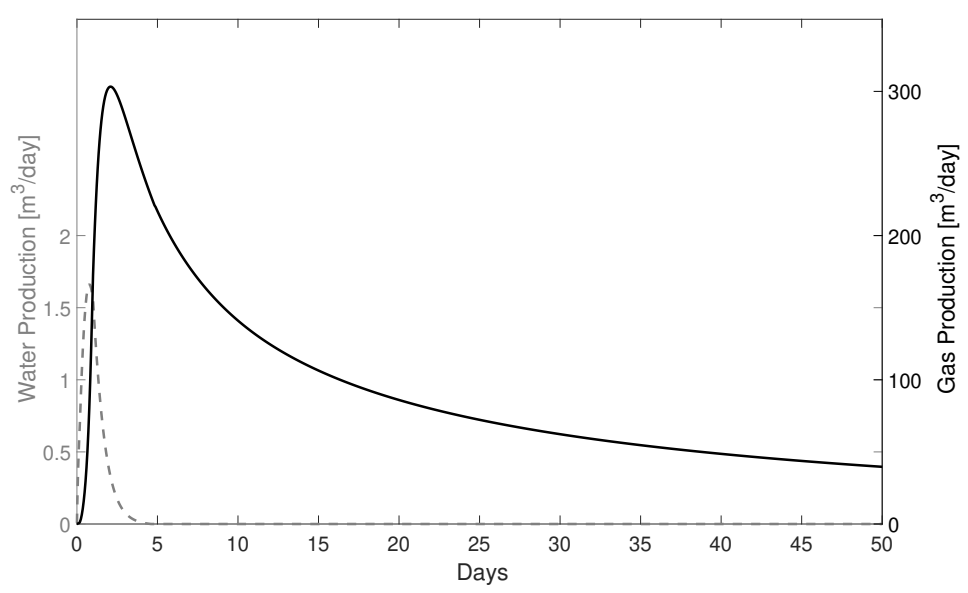

Figure 7: Reference case parameters: production curves.

Figure 8 shows the integration of the gas production with time to give the cumulative gas production curve. The total production tends to $8330 \mathrm{~m}^{3}$ (dotted line), which is the total volume of gas which can be recovered given the Langmuir's parameters.

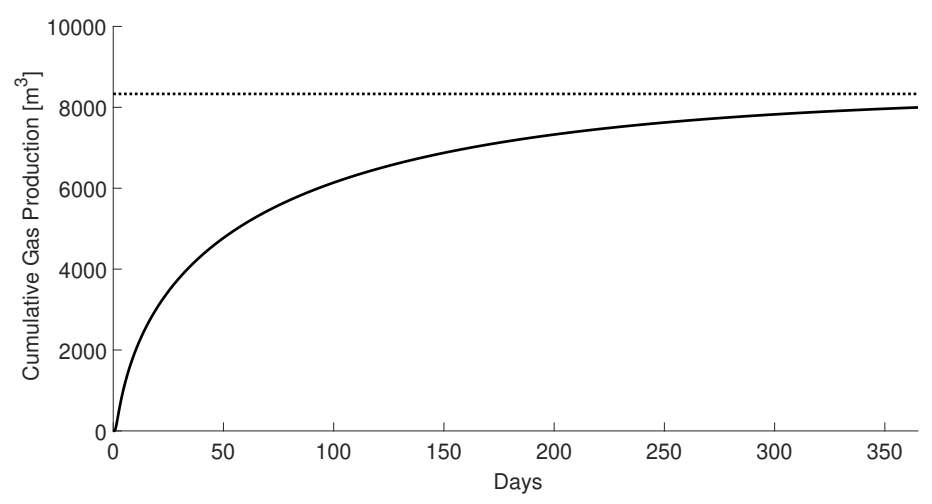

Figure 8: Reference case parameters: cumulative gas production 
These production curves hides the evolution of the permeability. As no macroscopic permeability is actually used (since there are no macroscopic constitutive laws), a trick is needed to obtain this information. In a conventional model, permeability links the fluxes and the hydraulic gradient. In our multiscale model, we still have a stiffness matrix to link fluxes and pressure gradient. However, this stiffness matrix component contains also the relative permeability evolution ${ }^{4}$ following desaturation. The intrinsic permeability is then given by:

$$
k=\frac{\mu_{w}}{k_{r w} \rho_{w}} \cdot K_{w w x}^{M}
$$

where $K_{w w x}^{M}$ is the macroscopic stiffness matrix component connecting the water flux towards the well and the corresponding pressure gradient. It is determined at each integration point from the miscrocale finite element computation.

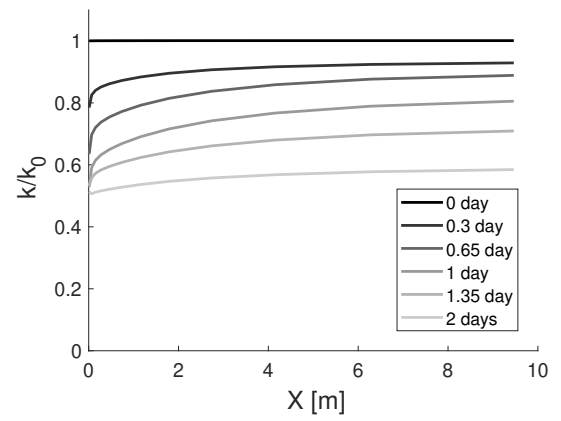

Figure 9: Reference case parameters: intrinsic permeability profiles at different times.

Figure 9 shows the profiles along the seam at different times of the intrinsic permeability evolution. The permeability is almost divided by two after two days with the parameters of this reference case. For this set of parameters, the model shows that, despite the shrinkage of the matrix which tends to increase the cleat aperture, the permeability is decreasing due to the increase of the effective stress following the depletion of the reservoir. The effective stress in the cleats has a larger effect than the shrinkage of the matrix in this case.

\subsection{Sensitivity analysis}

A sensitivity analysis is performed to get a better understanding of the model by evaluating the influence of the main parameters on the production curve. This analysis also highlights the hydro-mechanical couplings which are taken into account by the model. The sensitivity study is performed on the aperture $h$, the size of the REV, the

\footnotetext{
${ }^{4}$ The macroscopic relative permeability exists because the same retention curve is used for all the fractures (e.g. entry capillary pressure is independent of the aperture). However, the multiscale approach is not restricted to this case and calculating unused macroscopic variables is becoming increasingly meaningless.
} 
stiffness of the cleats $K_{n}$, the shrinkage coefficient $\beta_{\varepsilon}$, the Langmuir's parameters $V_{L}$ and $P_{L}$, the sorption time $\mathcal{T}$ and the retention curve $\left(p_{e}\right.$ and $\left.\lambda\right)$.

\subsubsection{Cleats aperture}

The parameter influencing most directly the response of the model is, with the number of cleats, the aperture of the cleats. The permeability is directly dependent on this aperture (Equations 38 and 43). Figure 10 shows the influence of the initial aperture of the cleats on the production curves. Higher the aperture, higher the production peak value. The ratios between the peak values are not straightforward since the transient behaviour plays a role in the shrinkage of the matrix. Moreover, the initial stiffnesses are initially not the same at $\sigma^{\prime}=2 M P a$ (Table 2). Indeed, the sensitivity study is carried out by modifying one parameter while all others remain equal. But modifying the initial aperture while keeping the same initial normal stiffness for the null stress actually leads to different initial stiffnesses for the given initial stress.

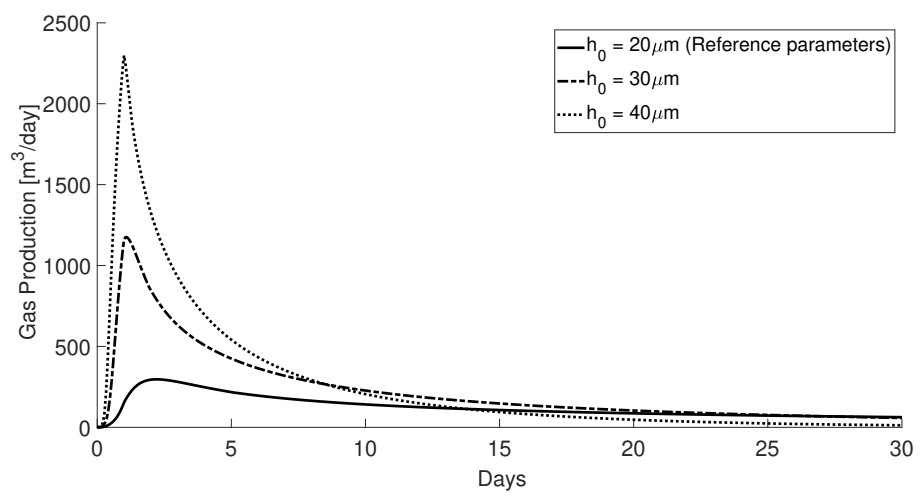

Figure 10: Influence of the cleat aperture on the gas production.

\begin{tabular}{l|l}
$\sigma^{\prime}=0 M P a$ & $\sigma^{\prime}=2 M P a$ \\
\hline \hline$h=20 \mu m$ & $h=10 \mu m$ \\
$K_{n}=100 G P a$ & $K_{n}=400 G P a$ \\
\hline$h=20 \mu m$ & $h=13.33 \mu m$ \\
$K_{n}=200 G P a$ & $K_{n}=450 G P a$ \\
\hline$h=20 \mu m$ & $h=18.18 \mu m$ \\
$K_{n}=1000 G P a$ & $K_{n}=1210 G P a$ \\
\hline$h=30 \mu m$ & $h=18 \mu m$ \\
$K_{n}=100 G P a$ & $K_{n}=278 G P a$ \\
\hline$h=40 \mu m$ & $h=26.67 \mu m$ \\
$K_{n}=100 G P a$ & $K_{n}=225 G P a$
\end{tabular}

Table 2: Initial apertures and normal stiffnesses.

\subsubsection{Cleats stiffness}

Figures 11 highlights the influence of the normal stiffness. Three different stiffnesses are compared, varying within an order of magnitude range. Higher the stiffness 
and higher the production peak since the aperture is less impacted by the increase of the effective stress following the reservoir depletion. Initial cleats apertures and normal stiffnesses at 2MPa are also presented in Table 2 using Equations (28) and (29).

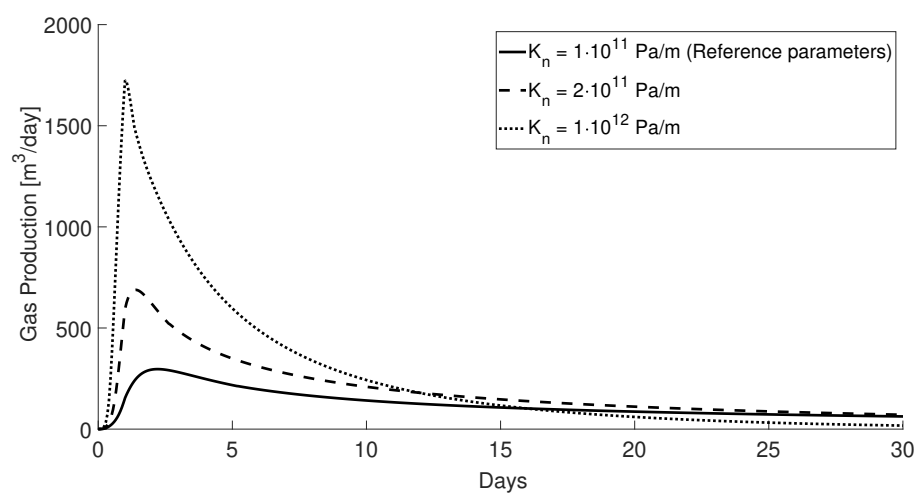

Figure 11: Influence of the cleat stiffness on the gas production.

\subsubsection{REV size (cleats density)}

The parameter now investigated is the REV size. For a given number of cleats, the permeability decreases with the increase of the REV size since the cleat density decreases. To analyse this cleat density effect, it is preferred to modify the REV size instead the number of cleats (Figure 12). It has the advantage of not increasing the computation cost. As the REV size is decreased, there is a decrease of the distance between cleats due to periodic conditions.

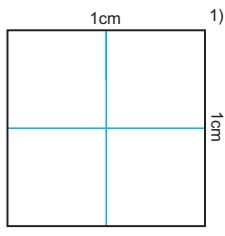

2)

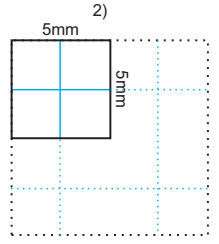

Figure 12: Cleat density geometries.

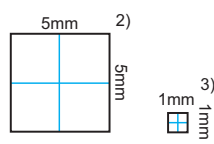

4)

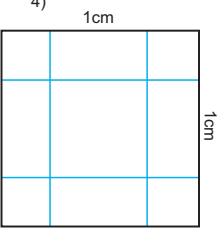

Figure 13 obviously shows that the reservoir is more quickly drained by the $1 \mathrm{~mm}$ REV (3) than the $5 \mathrm{~mm}$-REV (2) and the $1 \mathrm{~cm}$-REV (1) since the most fractured medium presents a greater permeability. The production obtained with the reference REV geometry (0) from Figure 4 is presented on the same figure, it matches the curve obtained 
with the $5 \mathrm{~mm}$-REV (2). Indeed, these two microstructures present the same density of horizontal fractures. The last geometry (4) corresponds to the extension of the $5 \mathrm{~mm}$ REV (2) over $1 \mathrm{~cm}$, it does not influence the response of the model either. The three curves (0), (2) and (4) are therefore superimposed. If a few simulations give the same result, they do not require the same CPU time. For instance, it took $2 h 30$ on a laptop ${ }^{5}$ to simulate 600 days using a 5000s time step with the REV (2) while it took $9 h$ to give the same results with the REV (4) using three times more nodes to discretize the microstructure. Correctly identifying the unit cell definitely saves time.

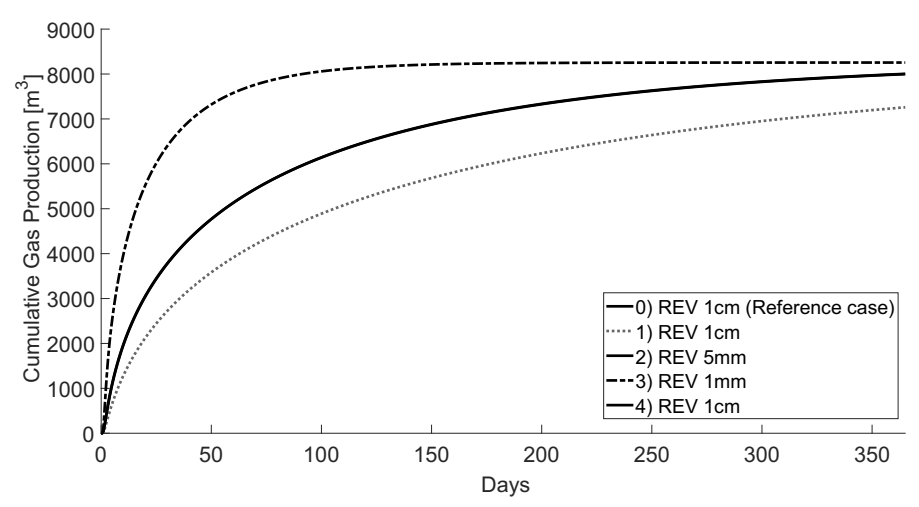

Figure 13: Influence of the cleat density on the cumulative production.

\subsubsection{Hydro-mechanical couplings}

The different simulations performed here aims to evaluate both the influence of the matrix shrinkage and the increase of the cleat effective stress on the gas production. On the first hand, the simulations performed with different shrinkage coefficients highlight the positive impact of the shrinkage coefficient on the permeability. On the other hand, comparing the simulation performed with a zero shrinkage coefficient with the one with a constant permeability highlights the negative impact of the increase of the cleat effective stress.

The exercise is first performed with the reservoir boundary conditions (Figure 14). It appears there is only a little effect of the matrix shrinkage on the permeability. Indeed, the decrease of volume is reflected more in the subsidence than in fracture aperture opening. Figure 15 shows the subsidence of the seam is linearly proportional to the linear swelling coefficient $\beta$. The subsidence observed for $\beta=0$ is due to the increase of effective stress following the depletion.

\footnotetext{
${ }^{5}$ Intel@ 6-core processor i7-8750H CPU @ 2.20GHz, RAM 16.0Go.
} 


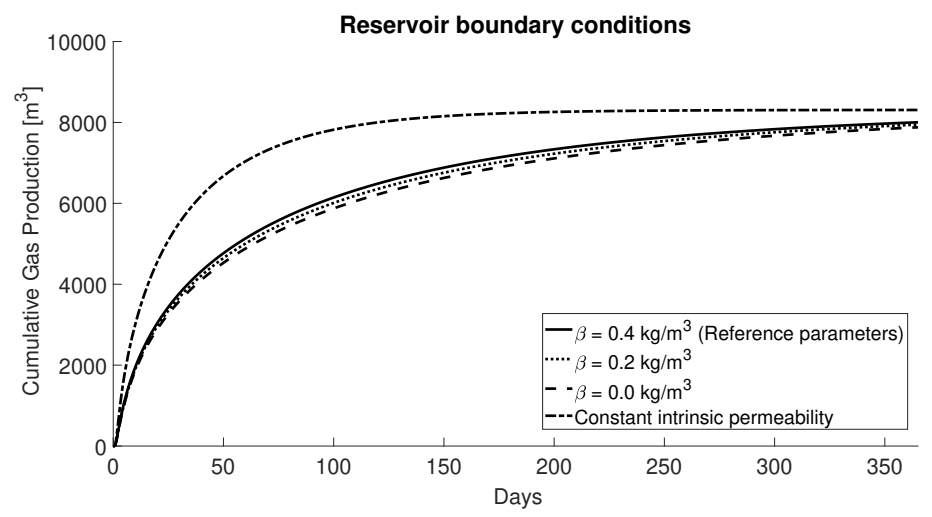

Figure 14: Influence of the hydro-mechanical couplings on the cumulative production.

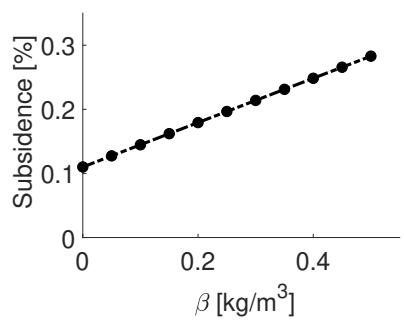

Figure 15: Influence of the shrinkage coefficient on the subsidence.

Considering constant volume conditions (Figure 16), the impact of the shrinkage is clearly more significant. For the chosen set of parameters with $\beta=0.4 \mathrm{~kg} / \mathrm{m}^{3}$, the negative impact of the effective stress is almost compensated by the positive impact of the shrinkage. It is likely these two boundary conditions, reservoir boundaries and constant volume, give a range of the production curve for a real reservoir. Note that considering the draining of the entire reservoir until the well pressure, it does not influence the total production. 


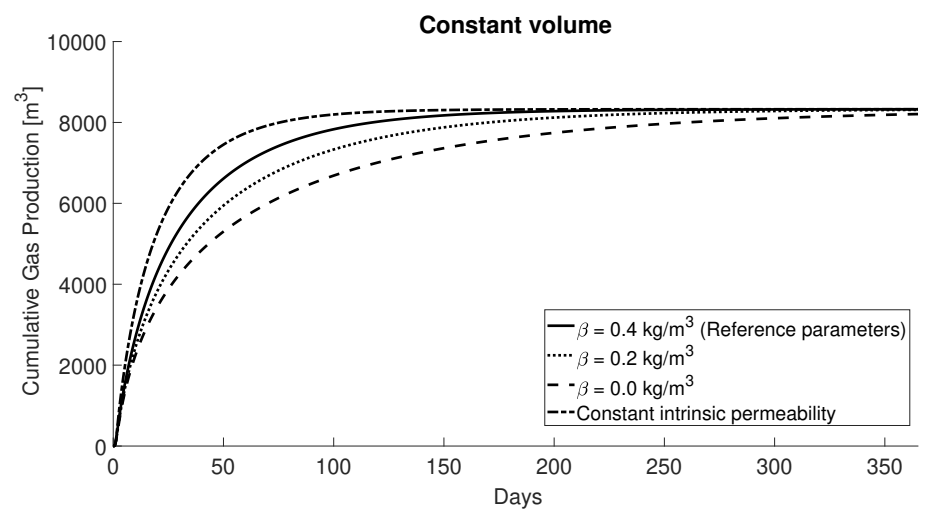

Figure 16: Influence of the hydro-mechanical couplings on the cumulative production.

Figure 17 demonstrates the positive influence of the shrinkage on the permeability evolution.

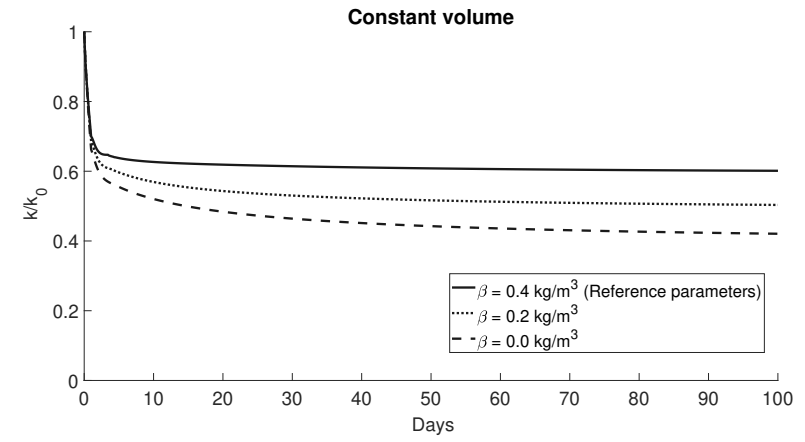

Figure 17: Influence of the shrinkage coefficient on the permeability at $x=6.6 \mathrm{~cm}$.

The question which arises now is which boundary condition should be actually used. Using a 2D REV geometry (in the same plane as the macroscale mesh), there is no other choice than meshing a cleat network separating the matrix blocks (otherwise the permeability is null). Such a structure is little impacted by the shrinkage if subsidence is allowed. However, the actual coal structure is likely to show attachment areas between the blocks while the fluid can bypass these obstacles via the third dimension. It means there are holding points that, locally, almost require volume conservation. Fractures could be therefore much more impacted by internal strains with reservoir boundary conditions than what is modelled with the 2D microsctructure. The two boundary cases applied on the 2D REV from Figures 4 and 12 should be therefore considered as two extreme cases for more complex fractures geometries. Depending on the application, gas recovery or gas injection, the most critical boundary conditions case are not the same. In practice, a detailed monitoring of the pressures and relative displacements of the seam would allow a proper quantification of the compaction process (Pineda et al., 2014) and see where it is situated in between the two boundary 
conditions.

\subsubsection{Langmuir's parameters}

Langmuir's parameters influence the total volume of gas produced from the reservoir since it is assumed the initial adsorbed gas volume is on the isotherm. For the same initial gas volume, different Langmuir's parameters also impact the desorption kinetics.

Figure 18 shows for instance that less gas is produced when $P_{L}=0.75 \mathrm{MPa}$ instead of $1.5 \mathrm{MPa}$. Indeed, despite the initial gas volume is higher with $0.75 \mathrm{MPa}$, the final volume is also higher and less gas is producible (Table 3 ).

\begin{tabular}{l|ccc} 
Parameters & $\begin{array}{c}V_{a d, 0}\left[\mathrm{~m}^{3}\right] \\
(p=3 \mathrm{MPa})\end{array}$ & $\begin{array}{c}V_{a d, f}\left[\mathrm{~m}^{3}\right] \\
(p=1 \mathrm{MPa})\end{array}$ & $\Delta V_{a d}\left[\mathrm{~m}^{3}\right]$ \\
\hline \hline$V_{L}=0.02 \mathrm{~m}^{3} / \mathrm{kg}$ & 20830 & 12500 & 8330 \\
$P_{L}=1.50 \mathrm{MPa}$ & & & \\
\hline$V_{L}=0.01 \mathrm{~m}^{3} / \mathrm{kg}$ & 10420 & 6250 & 4170 \\
$P_{L}=1.50 \mathrm{MPa}$ & & & 7150 \\
\hline$V_{L}=0.02 \mathrm{~m}^{3} / \mathrm{kg}$ & 25000 & 17850 & \\
$P_{L}=0.75 \mathrm{MPa}$ & & &
\end{tabular}

Table 3: Adsorbed gas volumes.

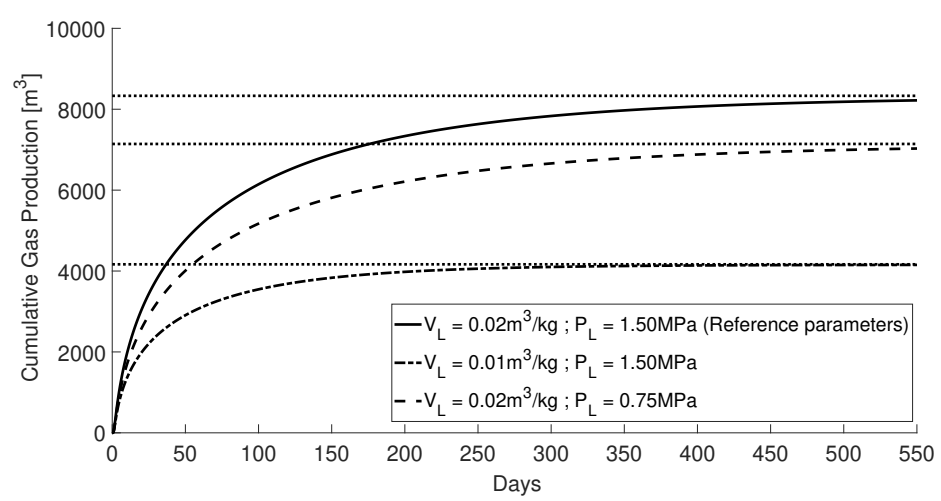

Figure 18: Influence of the Langmuir's parameters on the gas production.

\subsubsection{Sorption time}

Figure 19 highlights the influence of the sorption time on the production curve. The sorption time coefficient was introduced into the model to take into account the time required to diffuse through the matrix to reach the cleats. The greater the sorption time, the longer it takes to produce the gas. 


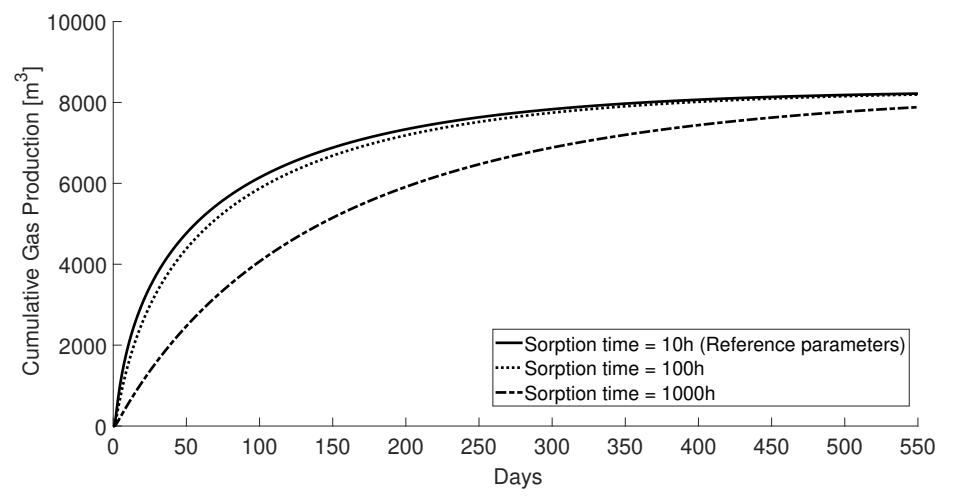

Figure 19: Influence of the sorption time on the gas production.

\subsubsection{Retention curve}

Finally, the last parameters tested are the parameters from the retention curve. Figure 20 shows that a greater gas entry value $p_{e}$ delays the production and a greater distribution index $\lambda$ speeds up the production. These behaviours are related to the water saturation degree. Figure 21 shows the saturation degree evolution close to the well for the different retention curves. A lower water saturation degree increases the gas permeability and therefore enhances the gas production.

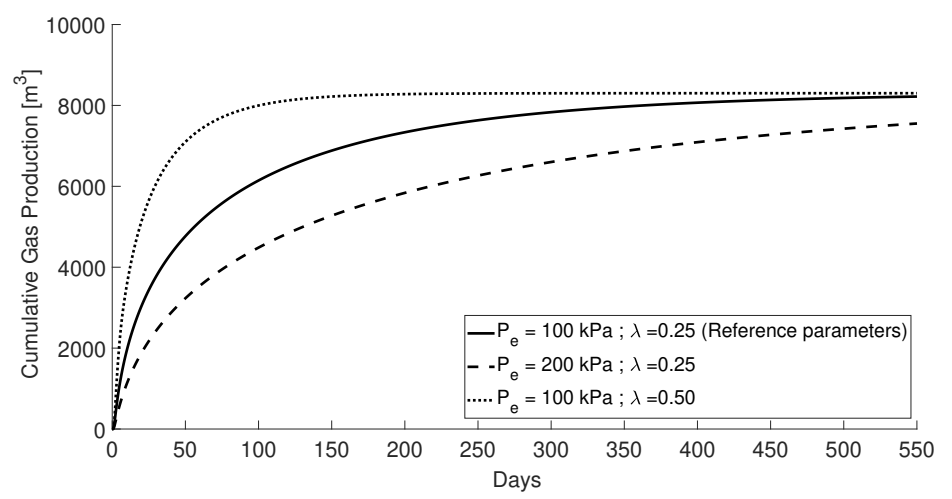

Figure 20: Influence of the parameters of the retention curve on the production. 


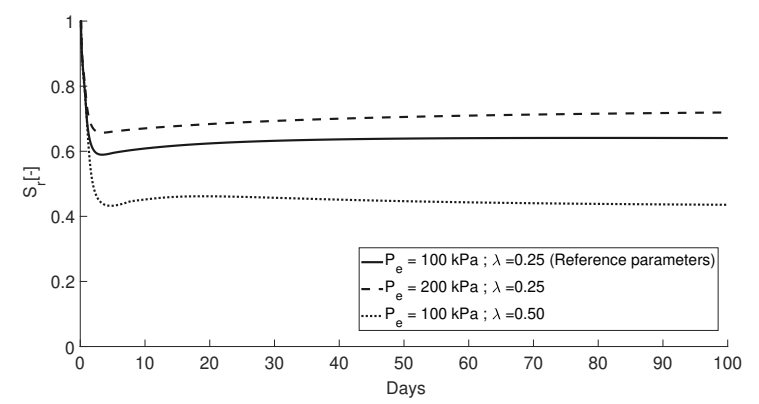

Figure 21: Saturation degree with time at $x=6.6 \mathrm{~cm}$ from the well for different retention curves.

\subsection{Horseshoe Canyon case}

The pressure and gas rate data presented by (Gerami et al., 2007) and taken up in the review of Pan and Connell on testing data (Pan and Connell, 2012) are used for a history matching exercise. Reservoir properties are listed in Table 4. The 300 meters of the reservoir are meshed with 20 macroscopic elements with a first element of $5 \mathrm{~cm}$ like in the reference case. Axisymmetric conditions are considered as for the synthetic reservoir. The REV geometry considered is that of 2D matrix blocks $1 \mathrm{~cm}$ wide. Model parameters used for this history matching exercise are given in Table 5.

\begin{tabular}{lr}
\hline Parameters & Values \\
\hline & \\
Initial gas pressure $(\mathrm{Pa})$ & $1.5 \mathrm{E} 6$ \\
Initial water pressure $(\mathrm{Pa})$ & $0 \mathrm{E} 6$ \\
Temperature $(\mathrm{K})$ & 289 \\
Coal density $\left(\mathrm{kg} / \mathrm{m}^{3}\right)$ & 1468 \\
Seam thickness $(\mathrm{m})$ & 8.99 \\
Langmuir volume $V_{L}\left(\mathrm{~m}^{3} / \mathrm{kg}\right)$ & 0.0092 \\
Langmuir pressure $P_{L}(\mathrm{~Pa})$ & $4.652 \mathrm{E} 6$ \\
& \\
\hline
\end{tabular}

Table 4: Reservoir parameters (Gerami et al., 2007).

\begin{tabular}{lr}
\hline Parameters & Values \\
\hline & \\
Reservoir radius $(\mathrm{m})$ & 282 \\
Overburden pressure $(\mathrm{Pa})$ & $5 \mathrm{E} 6$ \\
Matrix Young's modulus $E_{m}(\mathrm{~Pa})$ & $5 \mathrm{E} 9$ \\
Matrix Poisson's ratio $\mathrm{v}_{m}$ & 0.3 \\
Matrix width $\mathrm{w}(\mathrm{m})$ & 0.01 \\
Cleat aperture ${ }^{*} \mathrm{~h}(\mathrm{~m})$ & $107 \mathrm{E}-6$ \\
Cleat normal stiffness ${ }^{*} K_{n}(\mathrm{~Pa} / \mathrm{m})$ & $6.6 \mathrm{E} 9$ \\
Cleat shear stiffness $K_{s}(\mathrm{~Pa} / \mathrm{m})$ & $6.6 \mathrm{E} 9$ \\
Sorption time $\mathcal{T}$ (hours) & 1000 \\
Matrix shrinkage coefficient $\beta_{\varepsilon}\left(\mathrm{kg} / \mathrm{m}^{3}\right)$ & 0.5 \\
&
\end{tabular}

Table 5: Reservoir parameters used to calibrate the $F E^{2}$ model for the Horseshoe Canyon case. ${ }^{*}$ Given for a null stress.

The pressure at the well is given as input to the model and the production rate is observed as the response of the model to this loading. Figure 22 compares the data with 
the results of the simulation performed with parameters provided in Tables 4 and 5. To perform this simulation on a dry reservoir, only one degree of freedom is used at the macroscale, the gas degree of freedom. It means the simulation is performed at constant volume and the hydro-mechanical couplings are maximized at the microscale. Indeed, constant volume conditions are applied on the microscale by fixing the macroscale mechanical degrees of freedom. Fractures are therefore more impacted by the matrix shrinkage in these conditions. It takes only 2 minutes to simulate 800 days with a maximum time step of 10 days.

The fitting looks good overall. The simulation seems to overestimate a bit the production between 200 and 400 days. Actually, it appears that many well pressure points are clearly higher than the main trend during this period. It tends to reduce the production and it may partly explain the difference with the simulation.

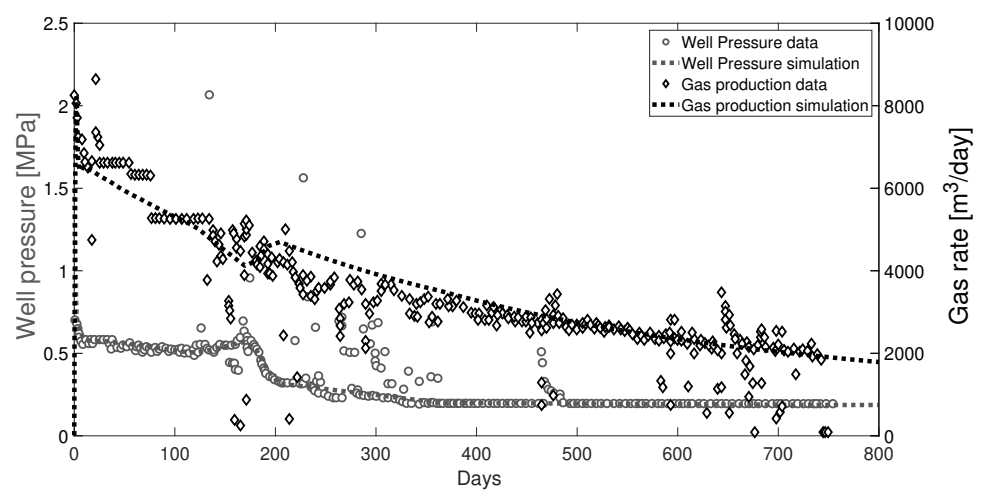

Figure 22: Matching exercise with the Horseshoe Canyon data.

Note the set of parameters that can be used to fit the data is not unique. The sensitivity analysis has shown the influence of the main parameters and they can be combined differently to obtain similar results. Actually, real predictive modelling would require validated parameters requiring a global experimental campaign on the material.

\section{Conclusions}

The localization and homogenization procedure of the $F E^{2}$ method was used to extend our cleat-scale model (Bertrand et al., 2019) from the laboratory scale to the reservoir scale. The model was implemented in the Lagamine code following the work of (Frey, 2010), (Marinelli, 2013) and (Van den Eijnden, 2015). Few contributions have been made in the purpose of coalbed methane production modelling (or reversely $\mathrm{CO}_{2}$ storage modelling). Mainly, a new degree of freedom for gas was introduced to deal with multiphase flow and a mechanism to account for swelling/shrinkage due to sorption/desorption was implemented. This model was applied to the modelling of one production well. The sensitivity study highlights the role of the main parameters on the response of the model to a pressure drop at the well. Parameters affecting the cleat 
aperture play of course a significant role in the production curves. But the influence of the boundary conditions on the hydro-mechanical couplings was also highlighted. Finally, the Horseshoe Canyon data were used for a history matching exercise. The determination of the whole set of independent parameters may be challenging in practice. It is also the reason why a simple elastic model was considered, as generally preferred in the petroleum industry.

In this paper, a deterministic approach is followed considering the cleat aperture is known. As we showed in the sensitivity analysis, different geometries give different permeabilities and thus different results. Coal generally presents cleats with apertures ranging from 3 to $40 \mu m$ (Laubach et al., 1998). In a next step, it could be interesting to follow a stochastic approach to account for the variability of all the parameters.

The $2 \mathrm{D}$ representative elementary volume means that the set of out-of-plane fractures is not taken into account in the permeability. There is actually nothing to prevent combining 2D at the macroscopic scale with 3D at the microscopic scale, but it would increase the computational cost. However, using a 3D-REV and 2D-axisymmetric conditions for the reservoir would overestimate the permeability since it would consider that the third set of fracture is radial with the wellbore. This combination is therefore not recommended for modelling a vertical well. Note that drilling technologies allow now to create horizontal wells. This case may be modelled with a macroscale mesh in plane strain state which is perpendicular to the well. The microstructure could be either in plane strain or more appropriately in 3D to account all the sets of cleats.

Compared to macroscale models, any fracture network could be used in the REV geometry of the multiscale model. Indeed, macroscale models are limited to a matchstick geometry while, despite simple unit cells were used in our modelling, this is not a limitation of the multiscale model. Moreover, as physical phenomena are written at the fracture scale, the formulation is sometimes simplified. For instance, there is no Biot's coefficient appearing in the effective stress equation since it is written at the cleat scale.

Compared to microscale models, transient effects are not directly considered at the microscale in the multiscale model. The REV boundary value problem is solved under steady state conditions but a sorption time $\mathcal{T}$ is taken into account for the storage term of the macroscale. The modelling of transient diffusive flows in the matrix would require the implementation of coupled elements with more than 4 nodes to avoid numerical oscillations. In addition, the pressure distribution at the microscopic scale at the end of each time step must be stored in memory to start the next time step.

So, new sophisticated tools are now available to model multiphase flow in naturally fractured medium after the implementation of this extended $F E^{2}$ model. The model was applied to methane recovery but it could also be applied to carbon storage. It can certainly prove useful for a wide range of applications, also outside coal-related applications. Indeed, the questions that have arisen on multiphase flows in a single fracture may also find applications in other media. 


\section{Nomenclature}

Greek Symbols

\begin{tabular}{|c|c|c|}
\hline $1 / \chi_{w}$ & Water compressibility & {$\left[M^{-1} L T^{2}\right]$} \\
\hline$\beta_{\varepsilon}$ & $\begin{array}{l}\text { Volumetric sorption-induced } \\
\text { strain coefficient }\end{array}$ & {$\left[M L^{-3}\right]$} \\
\hline$\delta_{i j}$ & Kronecker symbol & {$[-]$} \\
\hline$\Gamma$ & External surface of the REV & {$\left[L^{2}\right]$} \\
\hline$\kappa$ & $\begin{array}{l}\text { Geometric transmissivity func- } \\
\text { tion along the channel }\end{array}$ & {$\left[L^{3}\right]$} \\
\hline$\lambda$ & Cleat size distribution index & {$[-]$} \\
\hline$\lambda_{m}$ & $\begin{array}{l}\text { First Lamé parameter of the ma- } \\
\text { trix }\end{array}$ & {$\left[M L^{-1} T^{-2}\right]$} \\
\hline$\mu_{g}$ & Gas viscosity & {$\left[M L^{-1} T^{-1}\right]$} \\
\hline$\mu_{w}$ & Water viscosity & {$\left[M L^{-1} T^{-1}\right]$} \\
\hline$\Omega$ & Volume of the REV & {$\left[L^{3}\right]$} \\
\hline$\omega_{g}$ & Gas mass flux in the channel & {$\left[M T^{-1}\right]$} \\
\hline$\omega_{w}$ & Water mass flux & {$\left[M T^{-1}\right]$} \\
\hline$\Phi^{l}$ & $\begin{array}{l}\text { Geometric transmissivity of the } \\
\text { channel }\end{array}$ & {$\left[L^{2}\right]$} \\
\hline$\phi_{f}$ & Porosity from fractures & {$[-]$} \\
\hline$\rho_{c}$ & Coal density & {$\left[M L^{-3}\right]$} \\
\hline$\rho_{g}$ & Gas density & {$\left[M L^{-3}\right]$} \\
\hline$\rho_{g}^{A d}$ & Adsorbed gas density & {$\left[M L^{-3}\right]$} \\
\hline$\rho_{g}^{d}$ & Dissolved gas density & {$\left[M L^{-3}\right]$} \\
\hline$\rho_{w}$ & Water density & {$\left[M L^{-3}\right]$} \\
\hline$\rho_{g, s t d}$ & $\begin{array}{l}\text { Gas density at standard condi- } \\
\text { tions }\end{array}$ & {$\left[M L^{-3}\right]$} \\
\hline$\sigma_{i j}$ & Cauchy stress tensor & {$\left[M L^{-1} T^{-2}\right]$} \\
\hline$\tau$ & Shear stress & {$\left[M L^{-1} T^{-2}\right]$} \\
\hline$\theta$ & Angle & {$[-]$} \\
\hline
\end{tabular}




\begin{tabular}{|c|c|c|}
\hline$\varepsilon_{i j}$ & Strain tensor & {$[-]$} \\
\hline$\varepsilon_{v_{s}}$ & $\begin{array}{l}\text { Volumetric sorption-induced } \\
\text { strain }\end{array}$ & {$[-]$} \\
\hline \multicolumn{3}{|c|}{ Roman Symbols } \\
\hline$\left[K_{m m}\right]$ & Mechanical stiffness matrix & {$\left[M L^{-1} T^{-2}\right]$} \\
\hline $\mathcal{P}$ & Point with coordinates $x_{i}$ & \\
\hline $\mathcal{T}$ & Sorption time & {$[T]$} \\
\hline$f_{g_{i}}$ & Internal total flux of gas & {$\left[M L^{-2} T^{-1}\right]$} \\
\hline$f_{w_{i}}$ & Internal total flux of water & {$\left[M L^{-2} T^{-1}\right]$} \\
\hline$G_{m}$ & $\begin{array}{l}\text { Shear modulus of the matrix } \\
\text { blocks }\end{array}$ & {$\left[M L^{-1} T^{-2}\right]$} \\
\hline$h$ & Fracture aperture & {$[L]$} \\
\hline$h_{b}$ & Hydraulic fracture aperture & {$[L]$} \\
\hline$H_{g}$ & Henry's coefficient & {$[-]$} \\
\hline$K_{m}$ & $\begin{array}{l}\text { Bulk modulus of the matrix } \\
\text { blocks }\end{array}$ & {$\left[M L^{-1} T^{-2}\right]$} \\
\hline$K_{n}$ & Normal stiffness of the fracture & {$\left[M L^{-2} T^{-2}\right]$} \\
\hline$K_{n}^{0}$ & $\begin{array}{l}\text { Normal stiffness of the fracture } \\
\text { for zero-displacement }\end{array}$ & {$\left[M L^{-2} T^{-2}\right]$} \\
\hline$K_{s}$ & Shear stiffness of the fracture & {$\left[M L^{-2} T^{-2}\right]$} \\
\hline$k_{r g}$ & Relative permeability to gas & {$[-]$} \\
\hline$k_{r w}$ & Relative permeability to water & {$[-]$} \\
\hline$l_{R E V}$ & Size of the REV & {$[L]$} \\
\hline$M_{g}$ & Gas mass content & {$[M]$} \\
\hline$M_{w}$ & Water mass content & {$[M]$} \\
\hline$M_{g, f}^{d}$ & $\begin{array}{l}\text { Gas mass dissolved in the water } \\
\text { in the fracture }\end{array}$ & {$[M]$} \\
\hline$M_{g, f}^{g}$ & $\begin{array}{l}\text { Gas mass from the gas phase in } \\
\text { the fracture }\end{array}$ & {$[M]$} \\
\hline$M_{g, m}^{A d}$ & Gas mass adsorbed in the matrix & {$[M]$} \\
\hline$M_{m_{g}}$ & Gas molecular mass & {$\left[M N^{-1}\right]$} \\
\hline
\end{tabular}




\begin{tabular}{|c|c|c|}
\hline$p_{c}$ & Capillary pressure & {$\left[M L^{-1} T^{-2}\right]$} \\
\hline$p_{e}$ & Entry capillary pressure & {$\left[M L^{-1} T^{-2}\right]$} \\
\hline$p_{g}$ & Gas pressure & {$\left[M L^{-1} T^{-2}\right]$} \\
\hline$p_{g}^{*}$ & Virtual gas pressure & {$\left[M L^{-1} T^{-2}\right]$} \\
\hline$p_{g}^{f}$ & Fluctuation of gas pressure & {$\left[M L^{-1} T^{-2}\right]$} \\
\hline$P_{L}$ & Langmuir's pressure & {$\left[M L^{-1} T^{-2}\right]$} \\
\hline$p_{w}$ & Water pressure & {$\left[M L^{-1} T^{-2}\right]$} \\
\hline$p_{w}^{*}$ & Virtual water pressure & {$\left[M L^{-1} T^{-2}\right]$} \\
\hline$p_{w}^{f}$ & Fluctuation of water pressure & {$\left[M L^{-1} T^{-2}\right]$} \\
\hline$p_{g}^{A d}$ & Adsorbed gas pressure & {$\left[M L^{-1} T^{-2}\right]$} \\
\hline$q_{g}$ & Gas flux & {$\left[M L^{-2} T^{-1}\right]$} \\
\hline$q_{w}$ & Water flux & {$\left[M L^{-2} T^{-1}\right]$} \\
\hline$R$ & Universal gas constant & {$\left[M L^{2} N^{-1} \theta^{-1} T^{-2}\right]$} \\
\hline$s$ & Coordinate along the channel & {$[L]$} \\
\hline$S_{r}$ & Saturation degree & {$[-]$} \\
\hline$S_{r}^{*}$ & Normalized saturation & {$[-]$} \\
\hline$S_{r, r e s}$ & Residual saturation & {$[-]$} \\
\hline$T$ & Temperature & {$[\theta]$} \\
\hline$t$ & Time & {$[T]$} \\
\hline$t_{i}$ & Traction vector & {$\left[M L^{-1} T^{-2}\right]$} \\
\hline$u_{i}$ & Displacement vector & {$[L]$} \\
\hline$u_{i}^{f}$ & Fluctuation displacement field & {$[L]$} \\
\hline$v_{i}^{*}$ & Admissible virtual velocity field & {$\left[L T^{-1}\right]$} \\
\hline$V_{L}$ & Langmuir's volume & {$\left[L^{3} M^{-1}\right]$} \\
\hline$x_{i}$ & Coordinates vector & {$[L]$} \\
\hline \multicolumn{3}{|c|}{ Superscripts } \\
\hline$[.]^{F}$ & Quantity on the follow boundary & \\
\hline$[.]^{L}$ & Quantity on the lead boundary & \\
\hline
\end{tabular}


$[.]^{M} \quad$ Macroscale quantity

$[.]^{m} \quad$ Microscale quantity

[.] Time derivative

\section{Acknowledgements}

These researches were supported by the FNRS-FRIA and the WBI.

\section{References}

Bandis, S., Lumsden, A., and Barton, N. (1983). Fundamentals of rock joint deformation. 20(6):249-268.

Bertrand, F., Buzzi, O., and Collin, F. (2019). Cleat-scale modelling of the coal permeability evolution due to sorption-induced strain. International Journal of Coal Geology.

Bertrand, F., Cerfontaine, B., and Collin, F. (2017). A fully coupled hydro-mechanical model for the modeling of coalbed methane recovery. Journal of Natural Gas Science and Engineering, 46:307-325.

Charlier, R. (1987). Approche unifiée de quelques problèmes non linéaires de mécanique des milieux continus par la méthode des éléments finis (grandes déformations des métaux et des sols, contact unilatéral de solides, conduction thermique et écoulements en milieu poreux). $\mathrm{PhD}$ thesis, Université de Liège.

Chen, D., Pan, Z., Liu, J., and Connell, L. D. (2013). An improved relative permeability model for coal reservoirs. International Journal of Coal Geology, 109:45-57.

Connell, L. D., Lu, M., and Pan, Z. (2010). An analytical coal permeability model for tri-axial strain and stress conditions. International Journal of Coal Geology, 84(2):103-114.

Cui, X. and Bustin, R. M. (2005). Volumetric strain associated with methane desorption and its impact on coalbed gas production from deep coal seams. Aapg Bulletin, 89(9):1181-1202.

Dawson, G. and Esterle, J. (2010). Controls on coal cleat spacing. International Journal of Coal Geology, 82(3):213-218.

Feyel, F. and Chaboche, J.-L. (2000). $F E^{2}$ multiscale approach for modelling the elastoviscoplastic behaviour of long fibre sic/ti composite materials. Computer methods in applied mechanics and engineering, 183(3):309-330.

Frey, J. (2010). Modélisation multi-échelle de l'endommagement hydromécanique des roches argileuses. $\mathrm{PhD}$ thesis, Grenoble, INPG. 
Frey, J., Chambon, R., and Dascalu, C. (2013). A two-scale poromechanical model for cohesive rocks. Acta Geotechnica, 8(2):107-124.

Geers, M. G., Kouznetsova, V. G., and Brekelmans, W. (2010). Multi-scale computational homogenization: Trends and challenges. Journal of computational and applied mathematics, 234(7):2175-2182.

Gerami, S., Darvish, M. P., Morad, K., Mattar, L., et al. (2007). Type curves for dry cbm reservoirs with equilibrium desorption. In Canadian International Petroleum Conference. Petroleum Society of Canada.

Ghosh, S., Lee, K., and Moorthy, S. (1995). Multiple scale analysis of heterogeneous elastic structures using homogenization theory and voronoi cell finite element method. International Journal of Solids and Structures, 32(1):27-62.

Gilman, A. and Beckie, R. (2000). Flow of coal-bed methane to a gallery. Transport in porous media, 41(1):1-16.

Gu, F., Chalaturnyk, J., et al. (2005). Analysis of coalbed methane production by reservoir and geomechanical coupling simulation. Journal of Canadian Petroleum Technology, 44(10).

Gu, F. and Chalaturnyk, R. (2010). Permeability and porosity models considering anisotropy and discontinuity of coalbeds and application in coupled simulation. Journal of Petroleum Science and Engineering, 74(3):113-131.

Hill, R. (1965). A self-consistent mechanics of composite materials. Journal of the Mechanics and Physics of Solids, 13(4):213-222.

Kouznetsova, V., Brekelmans, W., and Baaijens, F. (2001). An approach to micromacro modeling of heterogeneous materials. Computational Mechanics, 27(1):3748.

Kouznetsova, V., Geers, M. G., and Brekelmans, W. M. (2002). Multi-scale constitutive modelling of heterogeneous materials with a gradient-enhanced computational homogenization scheme. International Journal for Numerical Methods in Engineering, 54(8):1235-1260.

Langmuir, I. (1918). The adsorption of gases on plane surfaces of glass, mica and platinum. Journal of the American Chemical society, 40(9):1361-1403.

Larsson, F., Runesson, K., and Su, F. (2010a). Computational homogenization of uncoupled consolidation in micro-heterogeneous porous media. International Journal for Numerical and Analytical Methods in Geomechanics, 34(14):1431-1458.

Larsson, F., Runesson, K., and Su, F. (2010b). Variationally consistent computational homogenization of transient heat flow. International Journal for Numerical Methods in Engineering, 81(13):1659-1686.

Laubach, S., Marrett, R., Olson, J., and Scott, A. (1998). Characteristics and origins of coal cleat: a review. International Journal of Coal Geology, 35(1):175-207. 
Lu, M. and Connell, L. D. (2007). A dual-porosity model for gas reservoir flow incorporating adsorption behaviour-part i. theoretical development and asymptotic analyses. Transport in porous media, 68(2):153-173.

Lu, S., Cheng, Y., and Li, W. (2016). Model development and analysis of the evolution of coal permeability under different boundary conditions. Journal of Natural Gas Science and Engineering, 31:129-138.

Marinelli, F. (2013). Comportement couplé des géo-matériaux: deux approches de modélisation numérique. $\mathrm{PhD}$ thesis, Université Grenoble Alpes.

Massart, T. J. (2003). Multi-scale modeling of damage in masonry structures. PhD thesis, Technische Universiteit Eindhoven.

Miehe, C., Schröder, J., and Schotte, J. (1999). Computational homogenization analysis in finite plasticity simulation of texture development in polycrystalline materials. Computer methods in applied mechanics and engineering, 171(3-4):387-418.

Moore, T. A. (2012). Coalbed methane: a review. International Journal of Coal Geology, 101:36-81.

Moulinec, H. and Suquet, P. (1998). A numerical method for computing the overall response of nonlinear composites with complex microstructure. Computer methods in applied mechanics and engineering, 157(1-2):69-94.

Nguyen, T. K., Combe, G., Caillerie, D., and Desrues, J. (2014). Fem × dem modelling of cohesive granular materials: numerical homogenisation and multi-scale simulations. Acta Geophysica, 62(5):1109-1126.

Nitka, M., Combe, G., Dascalu, C., and Desrues, J. (2011). Two-scale modeling of granular materials: a dem-fem approach. Granular Matter, 13(3):277-281.

Özdemir, I., Brekelmans, W., and Geers, M. (2008). Computational homogenization for heat conduction in heterogeneous solids. International journal for numerical methods in engineering, 73(2):185-204.

Palmer, I., Mansoori, J., et al. (1996). How permeability depends on stress and pore pressure in coalbeds: a new model. In SPE Annual Technical Conference and Exhibition. Society of Petroleum Engineers.

Pan, Z. and Connell, L. D. (2012). Modelling permeability for coal reservoirs: a review of analytical models and testing data. International Journal of Coal Geology, 92:144.

Paterson, L., Meaney, K., and Smyth, M. (1992). Measurements of relative permeability, absolute permeability and fracture geometry in coal. In Coalbed Methane Symposium, pages 19-21.

Pham, K., Kouznetsova, V. G., and Geers, M. G. (2013). Transient computational homogenization for heterogeneous materials under dynamic excitation. Journal of the Mechanics and Physics of Solids, 61(11):2125-2146. 
Pineda, J., Sheng, D., et al. (2014). Coal seam gas extraction and their potential effects on surface subsidence: an overview. In 7th International Congress on Environmental Geotechnics: iceg2014, page 370. Engineers Australia.

Schröder, J. (2014). A numerical two-scale homogenization scheme: the fe2-method. In Plasticity and Beyond, pages 1-64. Springer.

Schwerer, F., Pavone, A., et al. (1984). Effect of pressure-dependent permeability on well-test analyses and long-term production of methane from coal seams. In SPE Unconventional Gas Recovery Symposium. Society of Petroleum Engineers.

Seidle, J., Jeansonne, M., Erickson, D., et al. (1992). Application of matchstick geometry to stress dependent permeability in coals. In SPE rocky mountain regional meeting. Society of Petroleum Engineers.

Shi, J.-Q., Pan, Z., and Durucan, S. (2014). Analytical models for coal permeability changes during coalbed methane recovery: Model comparison and performance evaluation. International Journal of Coal Geology, 136:17-24.

Smit, R., Brekelmans, W., and Meijer, H. (1998). Prediction of the mechanical behavior of nonlinear heterogeneous systems by multi-level finite element modeling. Computer Methods in Applied Mechanics and Engineering, 155(1):181-192.

Somerton, W. H., Soylemezoglu, I., and Dudley, R. (1975). Effect of stress on permeability of coal. 12(5):129-145.

Su, F., Larsson, F., and Runesson, K. (2011). Computational homogenization of coupled consolidation problems in micro-heterogeneous porous media. International Journal for Numerical Methods in Engineering, 88(11):1198-1218.

Suquet, P. (1987). Elements of homogenization for inelastic solid mechanics. Homogenization techniques for composite media, 272:193-278.

Terada, K., Hori, M., Kyoya, T., and Kikuchi, N. (2000). Simulation of the multi-scale convergence in computational homogenization approaches. International Journal of Solids and Structures, 37(16):2285-2311.

Terada, K. and Kikuchi, N. (1995). Nonlinear homogenization method for practical applications. ASME applied mechanics division-publications-AMD, 212:1-16.

Van den Eijnden, A., Bésuelle, P., Chambon, R., and Collin, F. (2016). A fe2 modelling approach to hydromechanical coupling in cracking-induced localization problems. International Journal of Solids and Structures, 97:475-488.

Van den Eijnden, B. (2015). Multi-scale modelling of the hydro-mechanical behaviour of argillaceuous rocks. PhD thesis, Université Grenoble Alpes.

Van der Sluis, O., Schreurs, P., Brekelmans, W., and Meijer, H. (2000). Overall behaviour of heterogeneous elastoviscoplastic materials: effect of microstructural modelling. Mechanics of Materials, 32(8):449-462. 
Wei, X. R., Wang, G. X., Massarotto, P., Golding, S. D., Rudolph, V., et al. (2007). A review on recent advances in the numerical simulation for coalbed-methane-recovery process. SPE Reservoir Evaluation \& Engineering, 10(06):657-666. 